DRAFT VERSION JULY 30, 2018

Preprint typeset using LTEX style emulateapj v. 04/20/08

\title{
DIGGING INTO NGC 6334 I(N): MULTIWAVELENGTH IMAGING OF A MASSIVE PROTOSTELLAR CLUSTER
}

\author{
C.L. Brogan ${ }^{1}$, T.R. Hunter ${ }^{1}$, C.J. Cyganowski ${ }^{2}$, R.Indebetouw ${ }^{1,3}$, H. Beuther ${ }^{4}$, K.M. Menten ${ }^{5}$, S. Thorwirth $^{5,6}$ \\ Draft version July 30, 2018
}

\begin{abstract}
We present a high-resolution, multi-wavelength study of the massive protostellar cluster NGC 6334 I(N) that combines new spectral line data from the Submillimeter Array (SMA) and VLA with a reanalysis of archival VLA continuum data, 2MASS and Spitzer images. As shown previously, the brightest $1.3 \mathrm{~mm}$ source SMA1 contains substructure at subarcsecond resolution, and we report the first detection of SMA1b at $3.6 \mathrm{~cm}$ along with a new spatial component at $7 \mathrm{~mm}$ (SMA1d). We find SMA1 (aggregate of sources a, b, c, and d) and SMA4 to be comprised of free-free and dust components, while SMA6 shows only dust emission. Our 1"'5 resolution $1.3 \mathrm{~mm}$ molecular line images reveal substantial hot-core line emission toward SMA1 and to a lesser degree SMA2. We find $\mathrm{CH}_{3} \mathrm{OH}$ rotation temperatures of $165 \pm 9 \mathrm{~K}$ and $145 \pm 12 \mathrm{~K}$ for SMA1 and SMA2, respectively. We estimate a diameter of $1400 \mathrm{AU}$ for the SMA1 hot core emission, encompassing both SMA1b and SMA1d, and speculate that these sources comprise a $\gtrsim 800$ AU separation binary that may explain the previously-suggested precession of the outflow emanating from the SMA1 region. Compact line emission from SMA4 is weak, and none is seen toward SMA6. The LSR velocities of SMA1, SMA2, and SMA4 all differ by 1-2 $\mathrm{km} \mathrm{s}^{-1}$. Outflow activity from SMA1, SMA2, SMA4, and SMA6 is observed in several molecules including $\mathrm{SiO}(5-4)$ and IRAC $4.5 \mu \mathrm{m}$ emission; $24 \mu \mathrm{m}$ emission from SMA4 is also detected. Eleven water maser groups are detected, eight of which coincide with SMA1, SMA2, SMA4, and SMA6, while two others are associated with the Sandell et al. (2000) source SM2. We also detect a total of $83 \mathrm{Class}^{\mathrm{I} \mathrm{CH}_{3} \mathrm{OH} 44 \mathrm{GHz}}$ maser spots which likely result from the combined activity of many outflows. Our observations paint the portrait of multiple young hot cores in a protocluster prior to the stage where its members become visible in the near-infrared.
\end{abstract}

Subject headings: stars: formation — infrared: stars — ISM: individual (NGC $6334 \mathrm{I}(\mathrm{N}))$ - masers — techniques: interferometric

\section{INTRODUCTION}

The formation process of massive stars is an important but poorly-understood phenomenon. Because most regions that are forming massive stars lie at distances of several kiloparsecs, and most massive stars form in clusters, the identification of high mass protostars requires both good sensitivity and high angular resolution to resolve the continuum and line emission within young protoclusters. The advent of (sub)millimeter interferometers allows the study of deeplyembedded thermal gas emission at arcsecond angular resolution, a realm that has historically been accessible only via maser and $\mathrm{cm} \lambda$ continuum emission. Research that combines observations of dust, thermal gas and maser gas offers a more complete picture of the star formation process, as these phenomena potentially trace objects at different evolutionary stages.

NGC 6334 is a luminous $\left(>10^{6} \mathrm{~L}_{\odot}\right)$ and relatively nearby (1.7 kpc; Neckel 1978) region of massive star formation containing sites at various stages of evolution (Straw \& Hyland 1989). Matthews et al. (2008) have recently completed a comprehensive single dish study of the submillimeter dust properties of the whole complex with $\sim 14^{\prime \prime}$ resolution and find a total dust mass of $\sim 1.7 \times 10^{4} \mathrm{M}_{\odot}$. Recent work by Persi \& Tapia (2008) suggests that the distance to NGC 6334

\footnotetext{
Electronic address: cbrogan@ nrao.edu

${ }^{1}$ NRAO, 520 Edgemont Rd, Charlottesville, VA, 22903, USA

2 University of Wisconsin, Madison, WI, 53706, USA

${ }^{3}$ University of Virginia, Charlottesville, VA, 22903, USA

${ }^{4}$ MPIA Heidelberg, Germany

5 MPIfR Bonn, Germany

${ }^{6}$ I. Physikalisches Institut, Universität zu Köln, Germany
}

may be as close as $1.6 \mathrm{kpc}$, though we adopt $1.7 \mathrm{kpc}$ for ease of comparison with earlier work. The dust core "I(N)" was first detected at $1 \mathrm{~mm}$ by Cheung et al. (1978) and later at $400 \mu \mathrm{m}$ by Gezari (1982) toward the northern end of the complex.

In the past decade, direct evidence of protostars in the $\mathrm{I}(\mathrm{N})$ region were found in the form of Class I and II methanol masers (Kogan \& Slysh 1998; Walsh et al. 1998) and a faint $3.6 \mathrm{~cm}$ source (Carral et al. 2002). In a previous paper, we presented the first millimeter interferometric observations of $\mathrm{I}(\mathrm{N})$ with $\sim 1$. $^{\prime \prime} 5$ resolution which revealed a cluster of compact dust continuum cores likely to be a protocluster (Hunter et al. 2006, see also Figure 1). Subsequent subarcsecond resolution $7 \mathrm{~mm}$ continuum observations by Rodríguez et al. (2007) resolved the principal source SMA1 into several components, and detected possible counterparts to SMA4 and SMA6 (also see Carral et al. 2002). Our recent observations of the ammonia inversion transitions up to $(6,6)$ demonstrated the presence of compact clumps of hot thermal gas within this protocluster (Beuther et al. 2007). Although water masers are excellent tracers of high mass star formation, previous observations of this region have been limited to single dish data (Moran \& Rodriguez 1980). Likewise, most of the millimeter line studies of this region have been performed with single-dish telescopes. For example, Pirogov et al. (2003) imaged the $\mathrm{N}_{2} \mathrm{H}^{+}$(1-0) line with a beamsize of $40^{\prime \prime}$ and found significantly stronger emission toward $\mathrm{I}(\mathrm{N})$ than toward the more luminous massive protocluster (source I) that lies $\sim 1^{\prime}$ south of $\mathrm{I}(\mathrm{N})$. In addition, the $\mathrm{SiO}$ $(2-1)$ and (5-4) transitions show strong wing emission from -50 to $+40 \mathrm{~km} \mathrm{~s}^{-1}$ when observed with beamsizes of $58^{\prime \prime}$ and 
23", respectively (Megeath \& Tieftrunk 1999).

Recently, we reported $3 \mathrm{~mm}$ interferometric imaging of $\mathrm{I}(\mathrm{N})$, which revealed compact $\mathrm{CH}_{3} \mathrm{CN}$ (5-4) emission toward SMA1 along with a bipolar outflow seen in $\mathrm{HCN}(1-$ 0) (Beuther et al. 2008). In this paper, we present the details of our $1.3 \mathrm{~mm}$ Submillimeter Array ${ }^{7}$ (SMA) spectral line dataset, along with new NRAO Very Large Array ${ }^{8}$ (VLA) $\mathrm{H}_{2} \mathrm{O}$ maser and $44 \mathrm{GHz}$ Class $\mathrm{I} \mathrm{CH}_{3} \mathrm{OH}$ maser data. We have also re-analyzed archival VLA continuum data from $3.6 \mathrm{~cm}$ to $7 \mathrm{~mm}, 2 \mathrm{MASS}$ near-IR and Spitzer mid-IR IRAC and MIPS images. Together these data confirm the identification of NGC $6334 \mathrm{I}(\mathrm{N})$ as an actively forming massive protostellar cluster.

\section{OBSERVATIONS}

\subsection{Submillimeter Array (SMA)}

Our SMA observations were made with six antennas - in May 2004 in the compact configuration, and in May 2005 in the extended configuration. In both tracks, two pointings were observed: source I at $17^{\mathrm{h}} 20^{\mathrm{m}} 53.44^{\mathrm{s}},-35^{\circ} 47^{\prime} 02.2^{\prime \prime}$ and source $\mathrm{I}(\mathrm{N})$ at $17^{\mathrm{h}} 20^{\mathrm{m}} 54.63^{\mathrm{s}},-35^{\circ} 45^{\prime} 08.5^{\prime \prime}(\mathrm{J} 2000)$. Only the data for source $\mathrm{I}(\mathrm{N})$ are presented in this paper. The SMA receivers are double sideband SIS mixers with $2 \mathrm{GHz}$ bandwidth (Blundell et al. 1998). The local oscillator was tuned to provide $216.6-218.6 \mathrm{GHz}$ in LSB and $226.6-228.6 \mathrm{GHz}$ in USB, yielding a FWHP primary beam size of $\sim 55^{\prime \prime}$. The correlator was configured for 3072 channels per sideband with a uniform spectral resolution of $0.8125 \mathrm{MHz}$. The correlator sideband separation was about $18 \mathrm{~dB}$ at the time of these observations. Typical system temperatures were $200 \mathrm{~K}$. The projected baseline lengths ranged from 10 to $180 \mathrm{~m}$. A continuum image produced from these data was presented in Hunter et al. (2006), however in order to correct for a half-channel error in SMA velocity labeling discovered in November 2007, the data have been completely re-reduced.

The gain calibrators were the quasars NRAO $530\left(23^{\circ}\right.$ distant) and J1924-292 (27 distant). Bandpass calibration was performed with observations of Uranus (2004 data) and 3C279 (2005 data). Flux calibration is based on observations of Jovian satellites and regular SMA monitoring of the quasar flux densities. The data were calibrated in Miriad, then exported to AIPS for imaging; the two sidebands were reduced independently. The AIPS task UVLSF was used to separate the line and continuum emission. Self-calibration was derived from the continuum, and solutions were transferred to the spectral line data. After self-calibration, the continuum data from both sidebands were combined and imaged with both natural and uniform weighting in order to achieve the best surface brightness sensitivity (the former) and best angular resolution (the latter). The naturally weighted im-

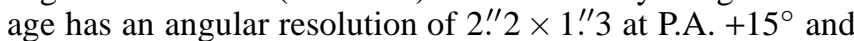
the uniformly weighted image has an angular resolution of $1{ }^{\prime \prime} 8 \times 0 .{ }^{\prime} 9$ at P.A. $+8^{\circ}$. The rms noise levels are 8.4 and 6.4 mJy beam ${ }^{-1}$ for the natural and uniform weighted images, respectively. The peak signal-to-noise of the naturally weighted continuum image has increased to 126 compared to 114 for the image presented in Hunter et al. (2006). The spectral line

\footnotetext{
7 The Submillimeter Array (SMA) is a collaborative project between the Smithsonian Astrophysical Observatory and the Academia Sinica Institute of Astronomy \& Astrophysics of Taiwan.

${ }^{8}$ The National Radio Astronomy Observatory is a facility of the National Science Foundation operated under agreement by the Associated Universities, Inc.
}

data were resampled to a spectral resolution of $1.1 \mathrm{~km} \mathrm{~s}^{-1}$ and imaged with natural weighting; the rms noise per channel is $180 \mathrm{mJy} \mathrm{beam}^{-1}$. The absolute position uncertainty of these data is estimated to be $0 . " 3$.

\subsection{Very Large Array (VLA) \\ 2.2.1. Water Maser Observations}

The $1.3 \mathrm{~cm}(22.235 \mathrm{GHz}) \mathrm{H}_{2} \mathrm{O}$ maser data were taken with the VLA on 2006 July 03 (project AH915) with online Hanning smoothing and one polarization (RR). The full bandwidth of the data is $3.125 \mathrm{MHz}\left(40 \mathrm{~km} \mathrm{~s}^{-1}\right.$ usable) and the channel separation and resolution is $24.4 \mathrm{kHz}\left(0.33 \mathrm{~km} \mathrm{~s}^{-1}\right)$. The absolute flux scale was set assuming a $22.235 \mathrm{GHz}$ flux density of 2.54 Jy for 3C286. Fast-switching was employed using J1717-337 and a cycle time of 2 minutes. The calibrator J1924-292 was used for bandpass calibration. The pointing model was updated once per hour during the observation. After flagging periods of poor phase coherence (due to weather), the on-source time is approximately 60 minutes. The FWHM primary beam of these data is $120^{\prime \prime}$ and the synthesized beam is $0 . " 79 \times 0 . ! 25$ at P.A. $=+7^{\circ}$.

It was necessary to remove the remarkably strong masers detected toward NGC 6334 I in the sidelobes (112" from the phase center of $\mathrm{I}(\mathrm{N})$ ) in order to achieve high dynamic range on the target masers in source $\mathrm{I}(\mathrm{N})$; source I masers were detected with peak intensities (uncorrected for primary beam attenuation) as high as $112 \mathrm{Jy}^{\text {beam }}{ }^{-1}$ despite their location near the first null. The removal was accomplished by selfcalibrating the data on the strongest source I channel, applying this calibration to the full line dataset, imaging both fields, subtracting the clean components from source I (only) in the $u-v$ plane (UVSUB), inverting the calibration (CLINV), reself-calibrating on the strongest $\mathrm{I}(\mathrm{N})$ maser channel, applying these new corrections and making the final $\mathrm{I}(\mathrm{N})$ line cube (this process is often called "peeling"). This procedure improved the dynamic range in the $\mathrm{I}(\mathrm{N})$ channels at the same velocities as the strong source I masers by a factor of $\sim 3$. The rms noise in a single channel is $6 \mathrm{mJy}^{\text {beam }}{ }^{-1}$. The absolute position uncertainty is approximately $0 .^{\prime \prime} 01$. Flux density measurements were made on an image corrected for primary beam attenuation.

\subsection{2. $44 \mathrm{GHz}$ Methanol Maser Observations}

The $7 \mathrm{~mm}(44.06941 \mathrm{GHz})$ Class $\mathrm{I} \mathrm{CH}_{3} \mathrm{OH}\left(7_{0,7}-6_{1,6}\right) \mathrm{A}^{+}$ maser transition was observed on 2008 February 18 with the VLA in CnB-configuration (project code AC904). Correlator mode $2 \mathrm{AB}$ was used with a velocity channel width of $0.17 \mathrm{~km} \mathrm{~s}^{-1}$. NGC6334I(N) was observed for 7.5 minutes (on-source) as a check source for a larger survey of massive protostellar candidates (Cyganowski et al. 2009). The gain, bandpass, and flux calibrators were J1717-337, J2253+161, and 3C286, respectively. Fast switching was employed with a cycle time of 2 minutes. The absolute positions are expected

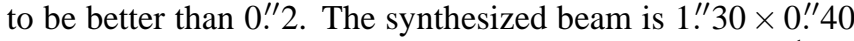
at P.A. $=-44^{\circ}$, and the velocity resolution is $0.33 \mathrm{~km} \mathrm{~s}^{-1}$ after Hanning smoothing. Due to the snapshot nature of these data, and consequent poor $u-v$ coverage, the noise is dynamic range limited in channels with strong maser emission. Channels with relatively weak maser emission have rms noise levels of $\sim 100 \mathrm{mJy}$ beam $^{-1}$, while it is up to $5 \times$ poorer in channels with strong maser emission (i.e. peak $>50 \mathrm{Jy}$ ).

2.2.3. Archival $7 \mathrm{~mm}, 1.3 \mathrm{~cm}$, and $3.6 \mathrm{~cm}$ Continuum Observations 


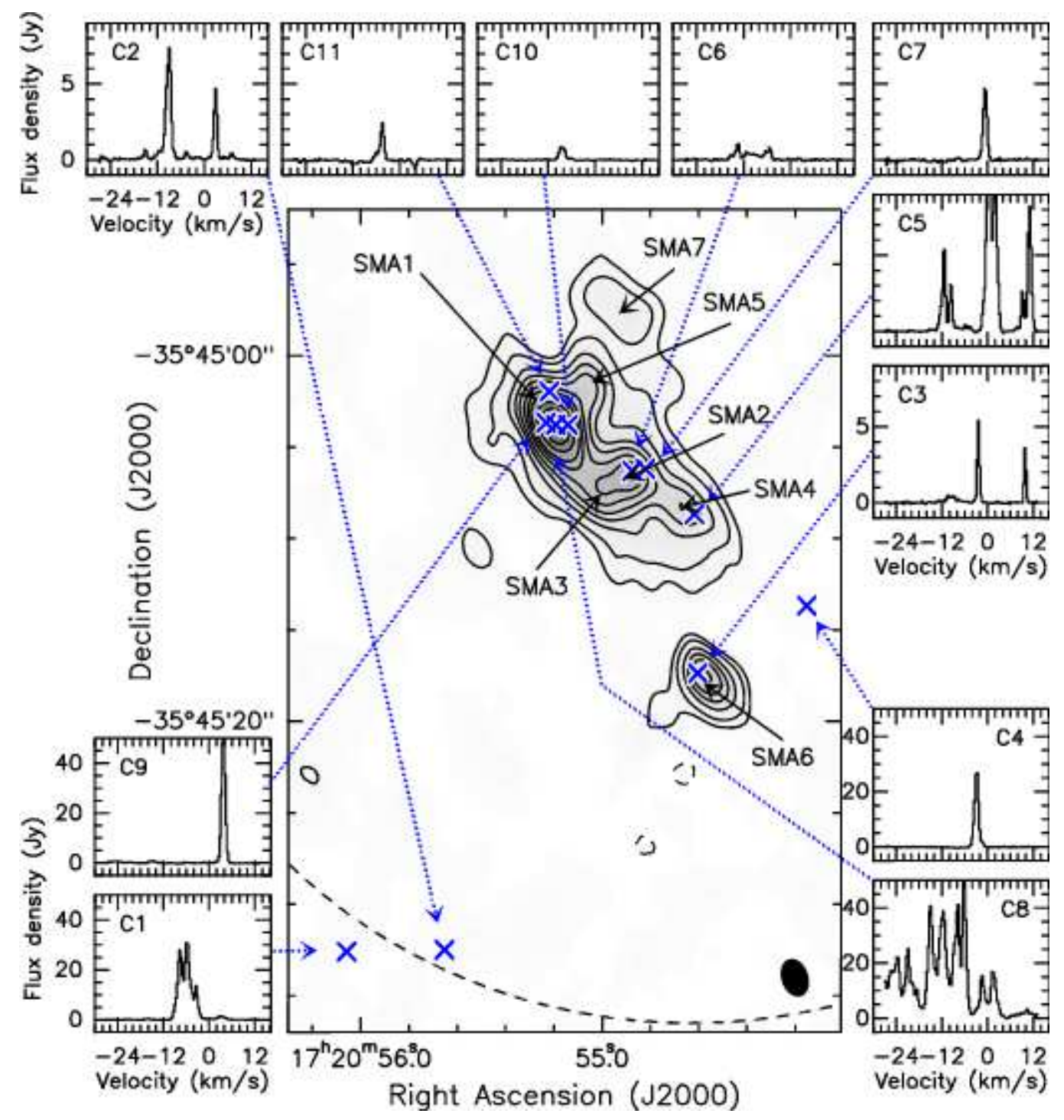

FIG. 1.- Composite image showing the naturally weighted SMA $1.3 \mathrm{~mm}$ continuum emission from $\mathrm{I}(\mathrm{N})$ with surrounding insets showing the VLA $\mathrm{H}_{2} \mathrm{O}$ $(22.235 \mathrm{GHz})$ maser spectra. The $1.3 \mathrm{~mm}$ continuum image has a resolution of $2 !^{\prime \prime} 2 \times 1{ }^{\prime \prime} 3\left(\mathrm{P} . \mathrm{A} .=+15^{\circ}\right)$ and the contour levels are $30(3.5 \sigma), 60,120,180,240$, $320,400,640,800,960 \mathrm{mJy}$ beam $^{-1}$. The blue crosses show the intensity weighted centroid positions of the water maser groups listed in Table 2 (derived from Table 3. The water maser spectra show the average emission from each group. The dashed arc indicates the SMA primary beam, and the dashed arc indicates the VLA primary beam at $22 \mathrm{GHz}$. The $1.3 \mathrm{~mm}$ image has not been corrected for primary beam attenuation.

We have re-reduced the archival VLA $7 \mathrm{~mm}$ and $1.3 \mathrm{~cm}$ continuum observations originally presented by Rodríguez et al. (2007, project codes AZ159 and AZ152, further details of these observations can be found in that paper). We achieved a significantly better rms noise in the $7 \mathrm{~mm}$ image $\left(210 \mu \mathrm{Jy}\right.$ beam $\left.^{-1}\right)$ than those authors report ( 320 $\mu \mathrm{Jy}$ beam $\left.^{-1}\right)$. This improvement is likely due to the fact that we simultaneously imaged a field toward source I which contains a strong ultracompact H II region ( $2^{\prime}$ south of $\mathrm{I}(\mathrm{N})$, see for example Hunter et al. 2006); images made without cleaning this $\mathrm{HII}$ region show significant sidelobes at the location of I(N). We also used the AIPS task VLANT to retrieve and apply post-observation antenna position corrections to the data, and used a model for the brightness distribution of the flux calibrator $3 \mathrm{C} 286$. The flux densities derived for the phase calibrator (J1720-355) are within $2 \%$ of those reported by Rodríguez et al. (2007). The angular resolution of the $7 \mathrm{~mm}$ data is $0 .{ }^{\prime \prime} 65 \times 0$." 44 , P.A. $=+25^{\circ}$. The $1.3 \mathrm{~cm}$ data were reduced in a similar manner and the rms noise is $64 \mu \mathrm{Jy} \mathrm{beam}^{-1}$, similar to that obtained by Rodríguez et al. (2007); the angular resolution is $0 .{ }^{\prime \prime} 38 \times 0 . \prime 27$, P.A. $=+19^{\circ}$. The $1.3 \mathrm{~cm}$ and $7 \mathrm{~mm}$ data show remarkable position agreement given that they were observed on different days - a testament to the accuracy of the VLA when reference pointing and fast switching are employed. We estimate that the absolute position accuracies for these two datasets are better than $0 .{ }^{\prime \prime} 05$.

We have also re-reduced the archival VLA $3.6 \mathrm{~cm}$ continuum observations originally presented by Carral et al. (2002, project code AM495, further details of the observing parameters can be found in that paper). The pointing center for these data is located $1.5^{\prime}$ south of source $\mathrm{I}(\mathrm{N})$ (the $3.6 \mathrm{~cm}$ FWHP is 5.4). These data were re-reduced as described above for the $7 \mathrm{~mm}$ and $1.3 \mathrm{~cm}$ data. In particular, a model for the brightness distribution of the flux calibrator (3C286) was employed - a method not available at the time of the original Carral et al. (2002) reduction. The average flux densities (between the two IFs) derived for the two phase calibrators, J1733-130 and $\mathrm{J} 1751-253$ are $6.67 \pm 0.03$ and $0.267 \pm 0.001$, respectively. 
In contrast Carral et al. (2002) obtained $7.6 \pm 0.2$ for J1733$130,14 \%$ higher than our more accurate result (a flux density for J1751-253 was not reported by these authors). After selfcalibration, the final image was made with baselines longer than $100 \mathrm{k} \lambda$ to minimize artifacts from the nearby (within $2^{\prime}$ ) extended H II regions "I" and "E", which fall within the VLA $3.6 \mathrm{~cm}$ FWHP primary beam. The resolution of the final $3.6 \mathrm{~cm}$ image of the $\mathrm{I}(\mathrm{N})$ region is 0 . $^{\prime} 51 \times 0 .{ }^{\prime} 20$, P.A. $=-1^{\circ}$ and the rms noise is $41 \mu \mathrm{Jy}$ beam $^{-1}$ after primary beam correction Carral et al. 2002, report an rms noise of $60 \mu \mathrm{Jy}_{\text {beam }}^{-1}$ but it is unclear whether they applied primary beam correction). The $3.6 \mathrm{~cm}$ data did not employ reference pointing or fast switching, and we estimate an absolute position accuracy for these data of $0 . " 3$. We note that there is a consistent $0 . " 2$ offset (to the SE) between the $3.6 \mathrm{~cm}$ data and the $1.3 \mathrm{~cm}$ and $7 \mathrm{~mm}$ data; we have not shifted the images to match.

\subsection{Archival Australia Telescope Compact Array (ATCA) $3.4 \mathrm{~mm}$ Observations}

We have re-imaged the $3.4 \mathrm{~mm}$ ATCA continuum data reported by Beuther et al. (2008). The rms noise is $3.3 \mathrm{mJy}^{\text {beam }}{ }^{-1}$ (after primary beam correction) and the resolution is 2 !" $3 \times 1$ ! $^{\prime}$, P.A. $=83^{\circ}$. Due to the small primary beam of the ATCA at this wavelength (FWHP 33" ), primary beam correction for this image is critical. This accounts for the apparent increase in rms noise compared to that reported by Beuther et al. (2008, $2.6 \mathrm{mJy}^{\text {beam }}{ }^{-1}$; our rms noise is $2.2 \mathrm{mJy} \mathrm{beam}^{-1}$ before primary beam correction); the peak signal-to-noise has increased by a factor of 1.3 . We estimate an absolute position accuracy for these data of $0 .{ }^{\prime \prime} 3$.

\subsection{Archival Infrared Data}

For near-infrared photometry, we analyzed archival 2 MASS $^{9}$ survey images (Skrutskie et al. 2006). We have also examined all of the Spitzer archival data for the $\mathrm{I}(\mathrm{N})$ region. Of the available IRAC data (Fazio et al. 2004), the data obtained in project 46 on 2004, August 2 by PI Fazio are by far the deepest (666 s). In this paper we use the PBCD images produced by pipeline version 14.0 Hunter et al. (a different version of these data were also presented in 2006). Since saturation in the $\mathrm{I}(\mathrm{N})$ region was not an issue, only the long integration (10.4 s) data are used. Unfortunately, of the available MIPS data, only the $24 \mu \mathrm{m}$ data are usable - the 70 and 160 $\mu \mathrm{m}$ data show tremendous striping and other artifacts. In this paper we utilize the $24 \mu \mathrm{m}$ data from the MIPSGAL Legacy survey (Carey et al. 2009).

We performed aperture photometry on all infrared images by defining irregular polygonal shapes (the "source" shape) surrounding each extended knot of emission (presumed to be shock fronts from one or more outflows, not point sources). A background is taken as the mode of pixel values in an scaled annulus, between 1.5 and 2 times radially expanded from the centroid of the source shape. These polygons are translated to each infrared image by their locations on the sky, so sample the identical regions even though the images have different pixel sizes and world coordinate systems. Fluxes were converted to Jy using the photometric information for each observatory.

\footnotetext{
9 Two Micron All Sky Survey, a joint project of the University of Massachusetts and the Infrared Processing and Analysis Center/California Institute of Technology, funded by the National Aeronautics and Space Administration and the National Science Foundation
}

\subsection{Caltech Submillimeter Observatory (CSO)}

Our $\mathrm{CSO}^{10}$ observations were obtained on 13 April 1993 using the facility $345 \mathrm{GHz}$ SIS receiver with lead junctions (Ellison et al. 1989) and a $500 \mathrm{MHz}$ AOS backend. Several on-the-fly maps of the CO 3-2 line were obtained on a $10^{\prime \prime}$ grid, providing near-Nyquist sampling of the $20^{\prime \prime}$ beam of the telescope at this frequency. At the end of each row, a designated off position $\left(17^{\mathrm{h}} 24^{\mathrm{m}} 56.06^{\mathrm{s}},-36^{\circ} 21^{\prime} 40.1^{\prime \prime} \mathrm{J} 2000\right)$ relatively free of $\mathrm{CO}$ emission was observed to provide the spectral baseline. After combining the maps, the total area covered was $4^{\prime} \times 4^{\prime}(25 \times 25$ grid $)$, with 30 seconds of on-source integration per point in the central portion containing source $\mathrm{I}$ and $\mathrm{I}(\mathrm{N})$, and 15 seconds per point on the periphery. The DSB receiver temperature was measured to be $320 \mathrm{~K}$ using the $y$-factor method, and the typical SSB system temperature was $1800 \mathrm{~K}$ at an elevation of $30^{\circ}$. The spectra have been corrected to the main beam brightness temperature scale using an efficiency of $68 \%$ as measured on Jupiter on the same night.

\section{RESULTS}

Our multiwavelength images reveal several sites of star formation activity within the $\mathrm{I}(\mathrm{N})$ region. In the following sections, we describe the variety of observed phenomena. In the following sections linear offsets are given with the $\gtrsim$ symbol to indicate that the measured values are lower limits since only the projected separation is available (a distance of 1.7 $\mathrm{kpc}$ is assumed).

\subsection{SMA $1.3 \mathrm{~mm}$ Continuum}

The naturally weighted $1.3 \mathrm{~mm}$ SMA continuum image of $\mathrm{I}(\mathrm{N})$ is presented in Figure 1 (center). This image is qualitatively similar to that presented in Hunter et al. (2006), but the calibration has been refined for improved signal-to-noise. These data resolve the presence of at least seven $1.3 \mathrm{~mm}$ "cores" in I(N). As pointed out in Hunter et al. (2006), this region has the hallmarks of a "protocluster", i.e. a region that will form a cluster of intermediate to massive stars. The integrated flux density within the $3 \sigma$ contour $\left(30 \mathrm{mJy} \mathrm{beam}^{-1}\right.$, see Fig. 10 of the naturally weighted $1.3 \mathrm{~mm}$ continuum image is $7.4 \pm 0.8 \mathrm{Jy}$ (this is 1.6 times higher than reported in Hunter et al. 2006); this estimate includes both statistical and $10 \%$ calibration uncertainties. Sandell (2000) find a $1.3 \mathrm{~mm}$ single dish flux density of $14.2 \pm 0.2 \mathrm{Jy}$, implying that we have recovered about $50 \%$ of the total flux with the SMA.

We have used the uniformly weighted image corrected for primary beam attenuation to fit the seven peaks identified in Figure 1 with a single 2-D Gaussian (using JMFIT in AIPS). The fitted positions and flux densities of the $1.3 \mathrm{~mm}$ cores are listed in Table 1 SMA1, SMA4, and SMA6 in particular, are not well-fit by a single Gaussian component, and instead appear to have a compact (unresolved, FWHM $<1^{\prime \prime}$ ) region of emission, embedded in a more extended though still compact (FWHM $<3^{\prime \prime}$ ) region. However, attempts to uniquely disentangle these two (or more) components with the current resolution and sensitivity were not successful. The JMFIT sizes listed in Table 1 are representative of the more extended (though still compact on the scale of the $\mathrm{I}(\mathrm{N})$ protocluster) component, and the integrated intensities and other quantities calculated from it ( $T_{b}$ and mass) are likely to be underestimates. These sizes while quite significant from a signal-tonoise perspective should be viewed as upper limits.

\footnotetext{
10 The Caltech Submillimeter Observatory $10.4 \mathrm{~m}$ is operated by Caltech under a contract from the National Science Foundation.
} 


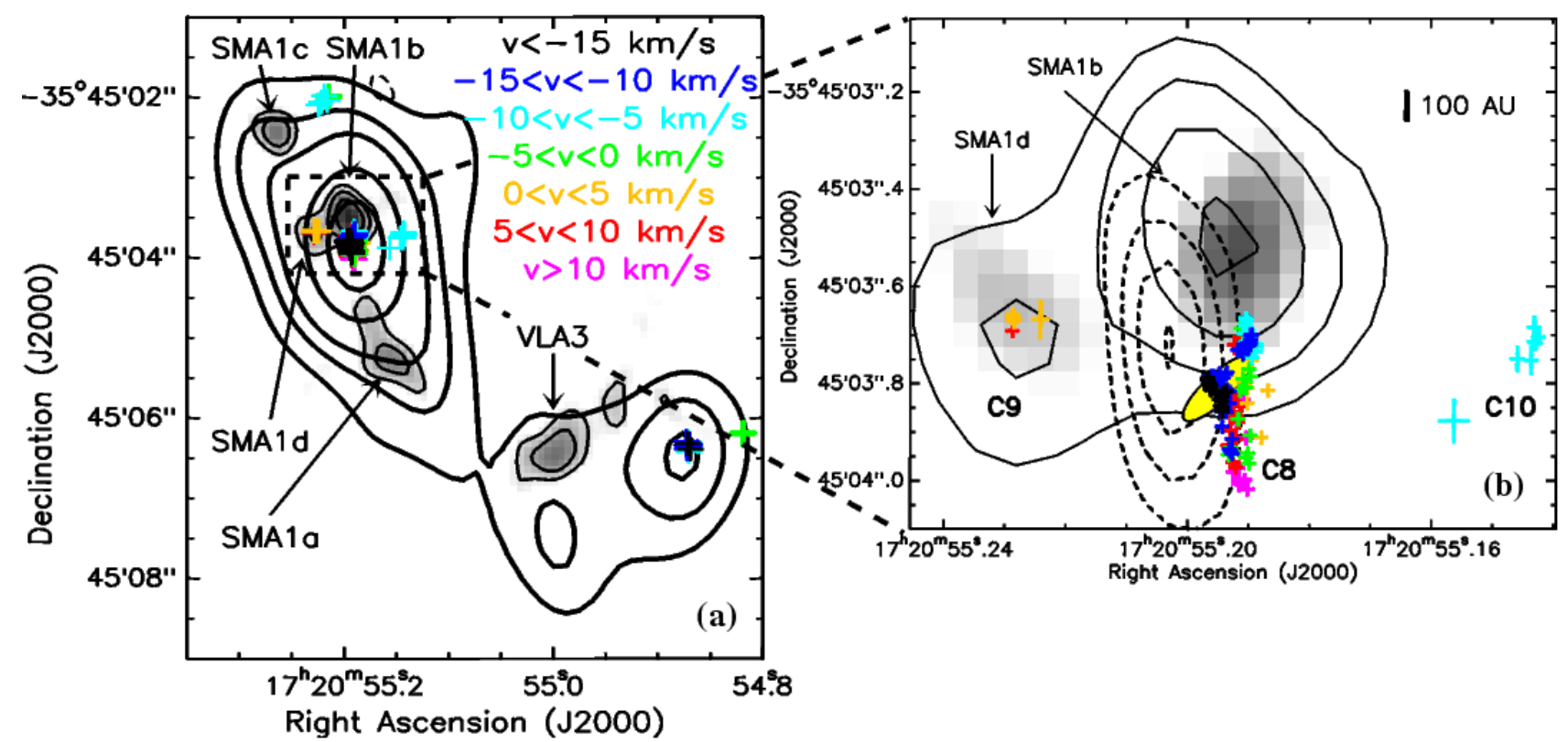

FIG. 2.-Zoomed multiwavelength images of I(N). (a) Zoom shows the SMA1, SMA2, and SMA3 regions with thick contours showing the uniformly weighted SMA 1.3 mm continuum image with contour levels of $170,250,330,490$, and 650 mJy beam $^{-1}$. Thin contours and greyscale show the VLA 7 mm continuum emission (contour levels are -0.6, 0.6, 0.8, 1.0, $1.2 \mathrm{mJy}_{\text {beam }}^{-1}$ ). The water maser positions are shown as colored crosses with a corresponding color coded velocity legend; note that the color scale was set using an assumed $-3 \mathrm{~km} \mathrm{~s}^{-1}$ systemic velocity for SMA1. (b) Zoom of the SMA1 region at $3.6 \mathrm{~cm}$ (dashed contours), $1.3 \mathrm{~cm}$ (greyscale), and $7 \mathrm{~mm}$ (solid contours). The $3.6 \mathrm{~cm}$ contour levels are $0.12,0.20,0.28$, and $0.36 \mathrm{mJy}$ beam ${ }^{-1}$, and the $7 \mathrm{~mm}$ contour levels are same as in (a). The water maser positions are also indicated with the same velocity color coding as (a). The position of the $44 \mathrm{GHz} \mathrm{CH}_{3} \mathrm{OH}$ maser is indicated by the yellow ellipse and it has a velocity of $-0.9 \mathrm{~km} \mathrm{~s}^{-1}$. For both types of maser symbol size indicates position uncertainty.

\subsection{ATCA $3.4 \mathrm{~mm}$ Continuum}

As first discussed by Beuther et al. (2008), the $3.4 \mathrm{~mm}$ continuum emission toward $\mathrm{I}(\mathrm{N})$ (not shown) is qualitatively similar to that at $1.3 \mathrm{~mm}$. With our new imaging of the original Beuther et al. (2008) data, we have been able to increase the signal-to-noise and reliably detect SMA4 at this wavelength. The fitted (JMFIT) parameters of the compact $3 \mathrm{~mm}$ continuum emission are given in Table 1

\subsection{VLA Water Masers and Continuum Emission}

The association of water masers with molecular outflows make them a good tracer of protostellar activity (Tofani et al. 1995; Hofner \& Churchwell 1996). We find strong $22 \mathrm{GHz} \mathrm{H}_{2} \mathrm{O}$ maser emission toward 11 distinct regions in NGC $6334 \mathrm{I}(\mathrm{N})$, listed in Table 2 in order of increasing declination. Masers that lie in close proximity to each other $\left(<1^{\prime \prime}\right)$ are collectively called by "group" names $\mathrm{C} 1$, C2...C11. The intensity weighted positions of these groups are listed in Table 2 and are plotted on the $1.3 \mathrm{~mm}$ continuum image in Figure 1, along with average spectra for each group (the position of each distinct $\mathrm{H}_{2} \mathrm{O}$ maser and its velocity are presented in Table 3, available in its entirety online). Eight of the groups lie within $2^{\prime \prime}$ of the $1.3 \mathrm{~mm}$ sources SMA1, SMA2, SMA4, and SMA6, whose positions are listed in Table 1. Two of the maser groups (C1 and $\mathrm{C} 2)$ lie near the SW edge of the SMA primary beam. These masers are coincident with the single-dish millimeter continuum source SM2, identified in JCMT submillimeter continuum images by Sandell (2000). Maser group C4 appears in a region with no compact millimeter continuum emission at the present sensitivity. Instead it lies at the southern edge of the $4.5 \mu \mathrm{m}$ nebula that extends to the southwest from SMA4 (Hunter et al. 2006, also see §3.6). More detailed phenomenology for the SMA $1.3 \mathrm{~mm}$ cores (except SMA5 and SMA7 which are weak and fairly diffuse) are given below.

\subsubsection{SMA1, SMA2, and SMA3 Regions}

Figure 2a shows a close-up view of the region from SMA1 through SMA3 in both 1.3 and $7 \mathrm{~mm}$ continuum. This region also contains $\mathrm{H}_{2} \mathrm{O}$ maser groups $\mathrm{C} 6$ through $\mathrm{C} 11$. Following Rodríguez et al. (2007) we call lower frequency continuum emission that appears to be associated with a particular SMA source by the "SMA name". If more than one source is associated with a $1.3 \mathrm{~mm}$ source, the SMA name is appended by $a, b, c$ etc. Lower frequency continuum emission that is not clearly associated with any particular SMA source is called "VLA" followed by the number of the closest SMA source. From the improvement in the $7 \mathrm{~mm}$ image quality over that shown in Rodríguez et al. (2007), several new features are present: (1) The $7 \mathrm{~mm}$ source SMA1b is resolved into two distinct sources separated by $\sim 0$." 45 ( $\gtrsim 800 \mathrm{AU}$, also see Figure $2 \mathrm{a}$ ), we shall refer to the eastern component as SMA1d; (2) SMA1a is elongated NE/SW and appears to point toward SMA1b; (3) Two new $7 \mathrm{~mm}$ features are detected to the north of SMA2 and SMA3, the stronger of the two is closest to SMA3 ( $\sim 1^{\prime \prime}$ north), and is thus called VLA3, while the other is barely above the $3 \sigma$ level and is not discussed further. The flux densities and positions of all of the continuum sources from $1.3 \mathrm{~mm}$ to $3.6 \mathrm{~cm}$ are provided in Table 1

Figure $2 \mathrm{~b}$ further zooms in on the continuum emission from SMA1b and SMA1d and the maser groups C8, C9, and C10. From this figure it is clear that SMA1d is also distinct from SMA1b at $1.3 \mathrm{~cm}$. From our reanalysis of the $3.6 \mathrm{~cm}$ data originally reported by Carral et al. (2002) we also detect SMA1b at this frequency. As mentioned in $\$ 2.2$ there appears to be a systematic $0 . / 2$ position offset of the $3.6 \mathrm{~cm}$ data which if removed would cause it to match the $1.3 \mathrm{~cm}$ and $7 \mathrm{~mm}$ position for SMA1b. Maser group C8, located $\sim 0$." 3 south of SMA1b, contains the brightest $\mathrm{H}_{2} \mathrm{O}$ maser in the whole region and is resolved into a linear north/south structure $\gtrsim 800 \mathrm{AU}$ in 


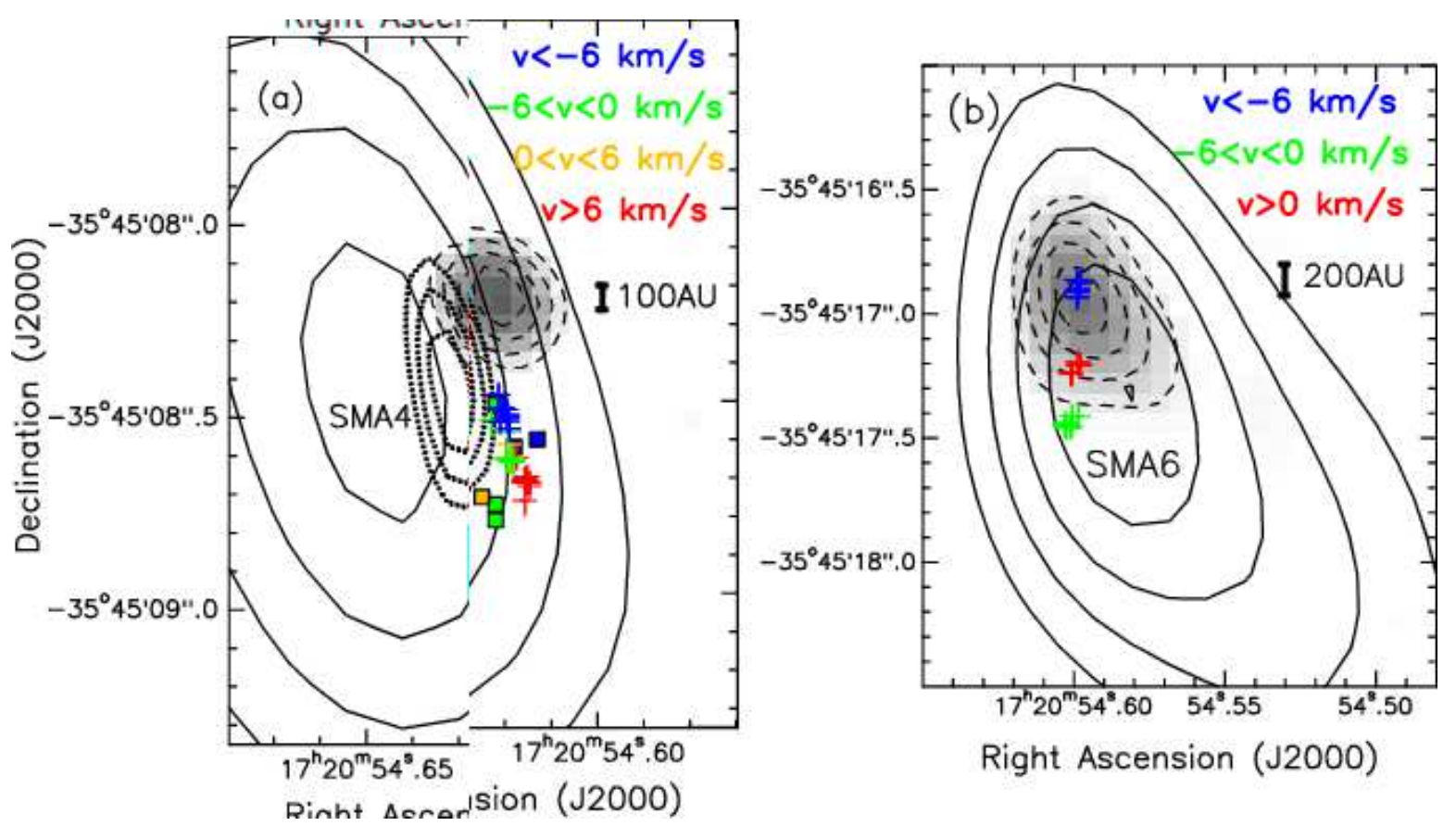

FIG. 3.- (a) Zoom of the SMA4 region with the grayscale and dashed contours showing the $1.3 \mathrm{~cm}$ continuum emission, dotted contours showing $3.6 \mathrm{~cm}$, and the solid contours showing the uniformly weighted $1.3 \mathrm{~mm}$ continuum. The $3.6 \mathrm{~cm}$ contours are $0.12,0.16$, and $0.20 \mathrm{mJy}^{-1}$ beam ${ }^{-1}$, the $1.3 \mathrm{~cm}$ contour levels are $0.19,0.25,0.31$, and $0.37 \mathrm{mJy}_{\text {beam }}^{-1}$, and the $1.3 \mathrm{~mm}$ contour levels are $100,120,140$, and $160 \mathrm{mJy} \mathrm{beam}^{-1}$. (b) Zoom of the SMA6 region with the greyscale and dashed contours showing $7 \mathrm{~mm}$ continuum emission and the solid contours showing the $1.3 \mathrm{~mm}$ continuum. The $7 \mathrm{~mm}$ contour levels are $0.6,0.8,1.0$, and $1.2 \mathrm{mJy}_{\text {beam }}{ }^{-1}$, and the uniformly weighted $1.3 \mathrm{~mm}$ contour levels are 100,140,180, 220, and $260 \mathrm{mJy}$ beam $^{-1}$. The cross symbols show the C5 (a) and $\mathrm{C} 3$ (b) water maser positions with $\mathrm{S} / \mathrm{N}$ ratio $>7$, color-coded by velocity. The filled square symbols in (a) show the location of $6.7 \mathrm{GHz} \mathrm{CH}_{3} \mathrm{OH}$ masers from Walsh et al. (1998), with same velocity coding as $\mathrm{H}_{2} \mathrm{O}$ masers.

length. The velocity structure of this linear group of masers is broad and complex, although the northern part is mostly blueshifted while the southern part is mostly redshifted with respect to the $\sim-3 \mathrm{~km} \mathrm{~s}^{-1}$ systemic velocity of SMA1 (see $\S 3.5$ for discussion of systemic velocities). These characteristics are similar to the brightest $\mathrm{H}_{2} \mathrm{O}$ maser component (C6) in the protocluster AFGL 5142 (Hunter et al. 1999) but on a somewhat larger physical scale. A $44 \mathrm{GHz}$ maser with a velocity of $\sim-0.9 \mathrm{~km} \mathrm{~s}^{-1}$ is also coincident with this linear $\mathrm{H}_{2} \mathrm{O}$ maser feature.

The redshifted and kinetically narrow $\mathrm{H}_{2} \mathrm{O}$ maser group $\mathrm{C} 9$ is coincident with SMA1d and contains the second brightest maser feature in $\mathrm{I}(\mathrm{N})$. A weak blueshifted group of masers (C10) lies $\gtrsim 1200$ AU southeast of SMA1b. Back on the larger scale of Fig. 2a, group C11 is another blueshifted narrow feature that lies $\gtrsim 1300$ AU northwest of SMA1c. Group C6 is $\gtrsim 400$ AU northwest of SMA2 and is blueshifted with respect to the systemic velocity of SMA2 $\left(\sim-5 \mathrm{~km} \mathrm{~s}^{-1}\right.$; see $\S 3.5$ ). Group $\mathrm{C} 7$ is a narrow but fairly strong component $\gtrsim 1500$ AU northwest of SMA2, and is near the systemic velocity.

\subsubsection{SMA4 Region}

Looking further south toward SMA4, the spatial/velocity structure of water maser group C5, as well as the $1.3 \mathrm{~mm}$ and 1.3 and $3.6 \mathrm{~cm}$ continuum emission in this region is shown in Figure $3 \mathrm{a}$. There are three distinct spatial clusters of $\mathrm{H}_{2} \mathrm{O}$ masers within the $\mathrm{C} 5$ group, corresponding to the three different velocity components. These three clusters of masers are oriented roughly north/south $\left(\right.$ P.A. $=17^{\circ}$ ) with blueshifted emission to the north, and redshifted emission to the south. The C5 group lies within $0 . \prime 4$ ( $\gtrsim 700 \mathrm{AU}$ ) of the $1.3 \mathrm{~mm}$ to $3.6 \mathrm{~cm}$ continuum emission peaks, just within the combined astronomical uncertainties of the data. The $\mathrm{C} 5$ group is also coincident with a cluster of Class II $6.7 \mathrm{GHz} \mathrm{CH}{ }_{3} \mathrm{OH}$ masers (Walsh et al. 1998; Caswell 2009) which have a similar velocity gradient. The 1.3 and $3.6 \mathrm{~cm}$ emission has been reported previously by Rodríguez et al. (2007) and Carral et al. (2002), respectively. We report the detection of SMA4 at $7 \mathrm{~mm}$ (not shown, see Table 1). As mentioned in $\S 2.2$ there appears to be a systematic offset of $0 .{ }^{\prime \prime} 2$ of the $3.6 \mathrm{~cm}$ data which if removed would cause it to match the $1.3 \mathrm{~cm}$ and $7 \mathrm{~mm}$ position. It is currently unclear if the maser and $1.3 \mathrm{~mm}$ offsets are significant (or simply due to absolute position uncertainties).

\subsubsection{SMA6 Region}

A zoomed in view of the SMA6 region is shown in Figure $3 \mathrm{~b}$. The $\mathrm{C} 3$ group of water masers is coincident with SMA6 and also has three distinct velocity clusters (see Fig. 3b). The kinematics of $\mathrm{C} 3$ are curious, with the three clusters lying along a roughly north-south line with systemic velocity to the south, then redshifted in the middle, and then blueshifted emission to the north. The $7 \mathrm{~mm}$ continuum peak toward SMA6 lies $\gtrsim 700$ AU north of the $1.3 \mathrm{~mm}$ peak, coincident with the northern blueshifted cluster of C3 masers. SMA6 is not detected at wavelengths longer than $7 \mathrm{~mm}$. The $1.3 \mathrm{~mm}$ morphology of SMA6 is suggestive of unresolved substructure in the north-south direction, and as mentioned in $§ 3.1$ is not well fit by a single Gaussian. Interestingly, although the $7 \mathrm{~mm}$ data has significantly better spatial resolution than the $1.3 \mathrm{~mm}$ data, both images show a similar morphology.

\subsection{VLA $44 \mathrm{GHz}$ Methanol masers}

Strong $44 \mathrm{GHz} \quad \mathrm{CH}_{3} \mathrm{OH}$ maser emission from NGC $6334 \mathrm{I}(\mathrm{N})$ was first detected in the single dish 


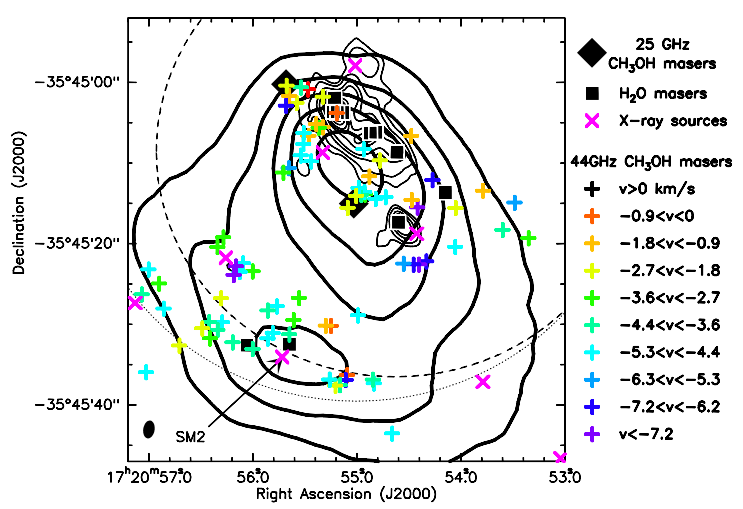

FIG. 4.- SMA $1.3 \mathrm{~mm}$ continuum contours of $\mathrm{I}(\mathrm{N})$ (thin black contours) with thick black contours showing SCUBA $450 \mu \mathrm{m}$ emission (G. Sandell, private communication). The $1.3 \mathrm{~mm}$ continuum image and contour levels are the same as Fig. 11 The SCUBA $450 \mu \mathrm{m}$ resolution is $8^{\prime \prime}$ and the contour levels are $60,80,100,120,160 \mathrm{Jy} \mathrm{beam}^{-1}$. The tip of the arrow for SM2 is the position reported by Sandell (2000). The colored crosses show the locations of $44 \mathrm{GHz}$ Class I methanol masers from this work. The locations of the water maser groups (also see Fig. 1) are shown by symbols and the $25 \mathrm{GHz}$ $\mathrm{CH}_{3} \mathrm{OH}$ masers reported by Beuther et al. (2005) are shown by symbols. The X-ray sources from Feigelson et al. (2009) are marked by magenta X's. The dashed arc indicates the SMA primary beam and the dotted arc indicates the VLA $44 \mathrm{GHz}$ primary beam. The $1.3 \mathrm{~mm}$ SMA continuum resolution is shown in the lower left.

survey of Haschick et al. (46" beam; 1990). Kogan \& Slysh (1998) resolved this emission into 23 spots using ten antennas of the VLA, but they were unable to determine accurate absolute positions. Our new observations with the full VLA provide a deeper more accurate view of the maser emission. By examining and comparing the details of the emission in each channel and from channel to channel, we have identified 83 individual maser spots. These are listed in Table 4, available in its entirety online. The values of $v_{\text {peak }}$ refer to the center velocity of the channel in which the peak emission occurs. The tabulated positions are the fitted position in the peak channel for each component. The peak brightness temperatures of these masers (see Table 4) lie far above the energy level of this transition $\left(65 \mathrm{~K}, 43.7 \mathrm{~cm}^{-1}\right)$, with values as high as $T_{b}=3.6 \times 10^{5} \mathrm{~K}$ with the current spatial resolution. While we have detected far more spots of emission, the spatial and velocity distribution of the Kogan \& Slysh (1998) spots are quite consistent with our data if one shifts the Kogan \& Slysh (1998) positions by $7.3^{\prime \prime}$ to the south-southwest. At the current level of sensitivity, no thermal emission was detected.

The maser positions are shown as velocity color-coded crosses in Figure 4.3. The peak maser velocities are in the range -8.49 to $-0.35 \mathrm{~km} \mathrm{~s}^{-1}$, close to the systemic velocity of the region. Figure 4.3 also shows a $450 \mu \mathrm{m}$ JCMT continuum image with $8^{\prime \prime}$ resolution from Sandell (2000). The masers are distributed over much of the area of dust emission, as delineated by the lowest $450 \mu \mathrm{m}$ contour. The masers can be divided into two main concentrations: the general area of the compact SMA continuum sources, and the area around the single-dish source SM2 (Sandell 2000). The former concentration contains a more diverse range of velocities, though no obvious trends in kinematics versus position are seen. It is notable that while these masers are clustered around $\mathrm{I}(\mathrm{N})$ $1.3 \mathrm{~mm}$ continuum sources, only one is positionally coincident with a compact SMA source (SMA1, see Fig. 2). The latter concentration towards the single dish continuum source SM2 is not associated with any $1.3 \mathrm{~mm}$ SMA continuum emission but this region lies outside the half-power point of the SMA data, significantly reducing the sensitivity in this region. Two $25 \mathrm{GHz}$ Class I $\mathrm{CH}_{3} \mathrm{OH}$ masers were also detected toward I(N) by Beuther et al. (2005), and both of these are coincident with $44 \mathrm{GHz}$ maser emission and have comparable velocities as shown in Fig. 4.3.

The position of the X-ray sources recently reported for this region (Feigelson et al. 2009) are also shown in Figure 4.3 The median photon energy of many of these X-ray sources is relatively hard $(\mathrm{E}>4 \mathrm{keV})$, suggesting deeply embedded sources. These authors have already noted the possible association of X-ray source 1454 with SMA7, and 1447 with SMA6 (although the latter is less likely given that its median photon energy is only $1 \mathrm{keV}$ ). With our new data, we note four additional possible associations: X-ray source 1470 is within 1. " 8 of the water maser component C2; and X-ray sources 1463, 1477 and 1487 each reside within a few arcseconds of a cluster of methanol masers. Source 1463 lies SSE of SMA1, just beyond the lowest millimeter contour. Source 1477 aligns with the shoulder of $450 \mu \mathrm{m}$ emission northeast of SM2, while source 1487 lies just east of the lowest $450 \mu \mathrm{m}$ contour

\subsection{SMA 1.3 Millimeter Molecular Line Data}

A total of 79 lines from 19 molecular species are detected toward SMA1 (Table 5) along with 14 unidentified features within the $4 \mathrm{GHz}$ of total bandwidth observed by the SMA. The spectra toward the SMA1 $1.3 \mathrm{~mm}$ continuum peak are shown in Figure 5. The lines were indentified using the CDMS (Müller et al. 2001), JPL (Pickett et al. 1998), and Splatalogue $^{11}$ spectral line databases. The large number of organic transitions detected, coupled with modest line brightness temperatures of $30 \mathrm{~K}$ suggests that this source harbors a moderate "hot core" (van Dishoeck \& Blake 1998). Many of the stronger lines detected toward SMA1 are also detected toward SMA2, though most are weaker, suggesting that this source also harbors a weak hot core. Other sources observed with similar linear resolution with this SMA setup have yielded both less diverse (S255N, Cyganowski et al. 2007), as well as much richer hot core spectra (NGC6334I, Brogan et al., in prep). The broadband SEST single-dish molecular line survey toward NGC 6334I and I(N) also shows that while source $\mathrm{I}(\mathrm{N})$ exhibits significant hot core emission, source I shows a much richer array of molecular line species along with stronger emission (Thorwirth et al. 2003).

\subsubsection{Extended Molecular Line Emission}

Many of the lines from low excitation states $\left(E_{l}<70 \mathrm{~cm}^{-1}\right)$ show emission extending to the NE and SW of the SMA continuum emission (see Figure 6). This emission is clearly tracing one or more outflows emanating from the protocluster. Interpretation of the extended emission is complicated by the fact that the SMA data are not sensitive to smooth emission on sizescales $\gtrsim 20^{\prime \prime}$ (due to spatial filtering by the interferomter). Generally, the emission to the northeast is red-shifted, while that to the southwest is blueshifted, but the kinematics become very confused in the southern part of $\mathrm{I}(\mathrm{N})$ with both red and blueshifted emission present. $\mathrm{SiO}$ in particular shows a striking bipolar appearance centered on SMA1. Channel maps of $\mathrm{SiO}$ (Figure $7 \mathrm{~A}$ ) reveal the complex velocity field of the extended emission. A lobe of molecular material also extends westward from SMA4 at low, but blueshifted veloci-

\footnotetext{
${ }^{11} \mathrm{http} / / /$ www.cv.nrao.edu/php/aremijan/splat_beta/
} 

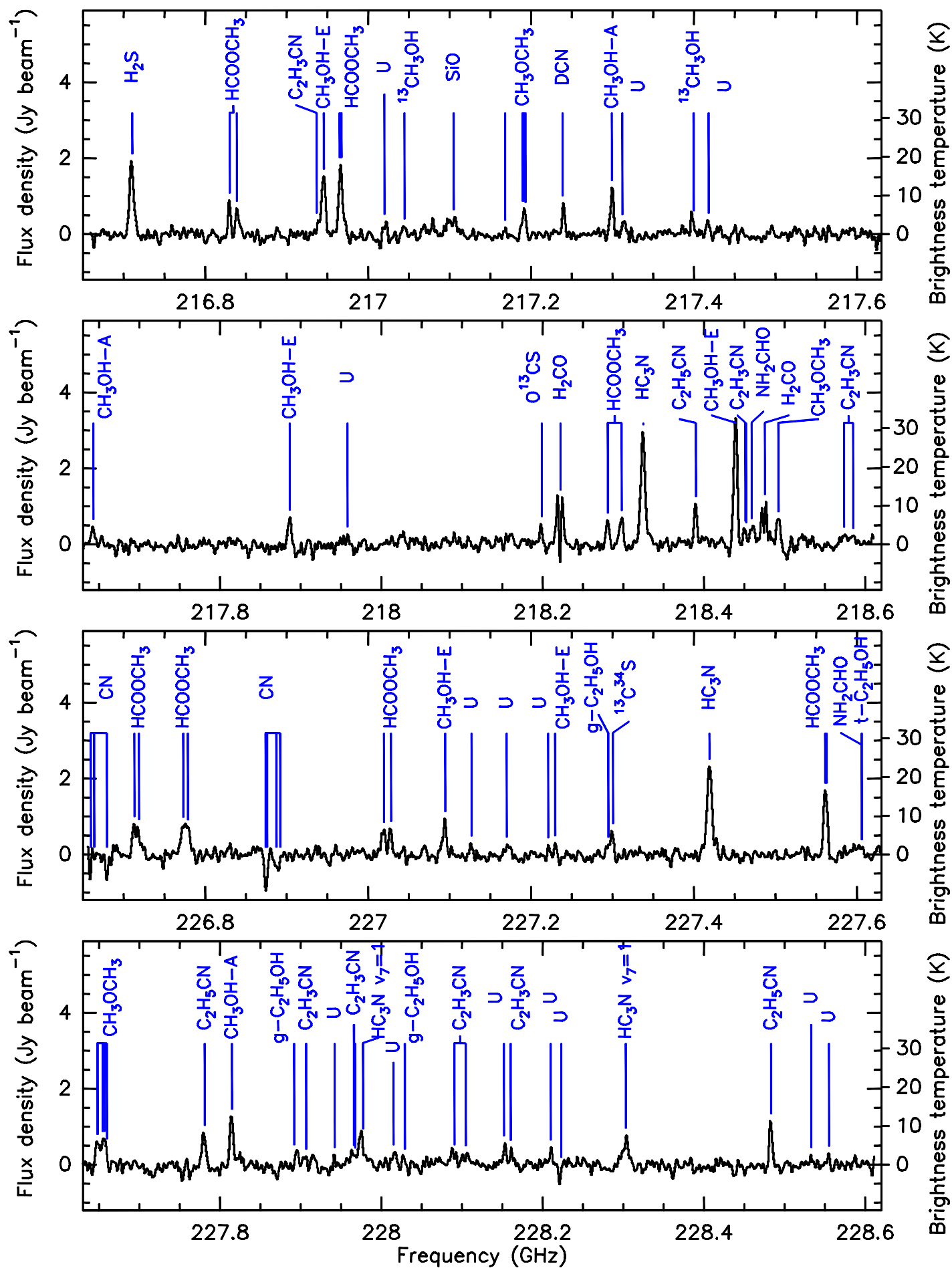

FIG. 5. - SMA spectra of the upper and lower sidebands from the $1.3 \mathrm{~mm}$ continuum peak of NGC6334I(N)-SMA1. The spectra have been Hanning smoothed.

ties in $\mathrm{SiO}$ and several other species shown in Fig. 6 $\left(\mathrm{H}_{2} \mathrm{CO}\right.$, $\mathrm{CH}_{3} \mathrm{OH}$, and $\mathrm{H}_{2} \mathrm{~S}$ for example).

A more compact $\mathrm{SiO}$ velocity gradient also appears to be centered on SMA6. CSO CO(3-2) spectra with $20^{\prime \prime}$ resolution show that the broad line wings toward SMA6 are of similar magnitude to those seen toward SMA1 (see Fig. 7b), suggesting both objects harbor outflows. The integrated red and blue-shifted emission around SMA6 is shown in detail in Figure 7t, and is more or less centered on the $\mathrm{CN}$ and $\mathrm{H}_{2} \mathrm{CO}$
$\left(E_{l}=7 \mathrm{~cm}^{-1}\right)$ absorption velocity of $\sim-4 \mathrm{~km} \mathrm{~s}^{-1}$ (see $\left.\$ 3.5 .2\right)$. This figure also shows that the sense of the $\mathrm{SiO}$ gradient is reflected in the position and velocity of the $44 \mathrm{GHz}$ methanol masers surrounding SMA6. However, on smaller scales the C3 group of water masers is oriented in a more north-south direction (Fig. 3b). It is unclear how the curious kinematics of the C3 water masers (from south to north: systemic, then red, then blueshifted features) fits in with the compact NE-SW outflow. 


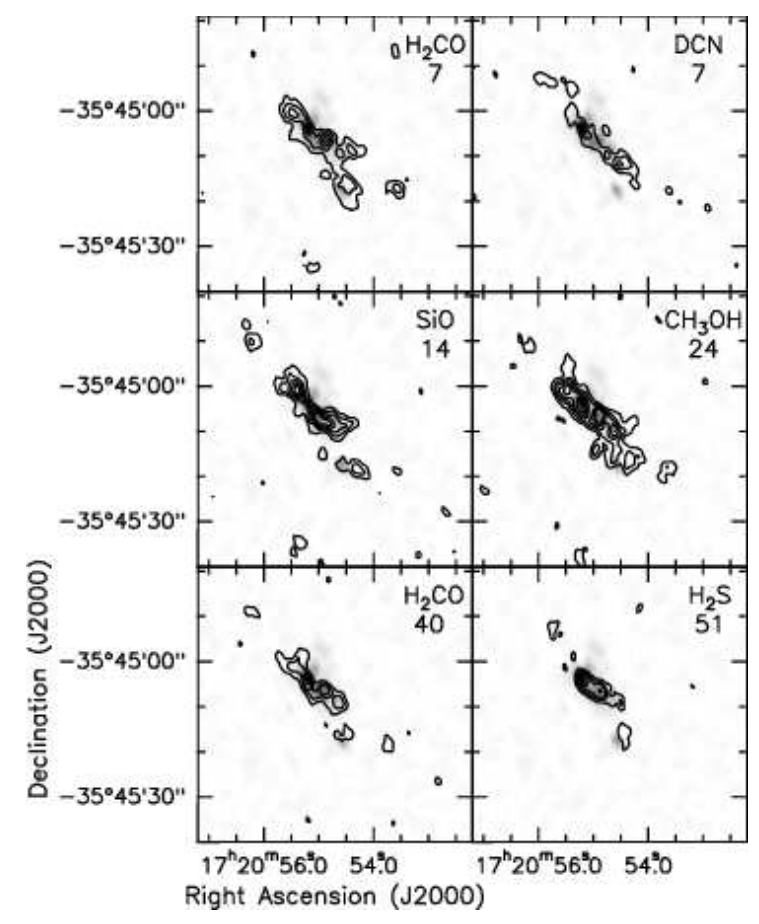

FIG. 6. - In each panel, the colorscale is the $1.3 \mathrm{~mm}$ continuum and the contours show the integrated intensity of the indicated spectral lines. The dashed circle is the FWHP of the SMA primary beam; these images have not been corrected for primary beam attenuation. The contour levels are $\mathrm{H}_{2} \mathrm{CO}\left(3_{0,3}-2_{0,2}\right): 4.35,7.25,10.15,13.05 \mathrm{Jy} \mathrm{km} \mathrm{s}^{-1}$; DCN: $1.77,2.95,4.13$ Jy km s${ }^{-1}$; SiO: 6, 10, 14, 18, $22 \mathrm{Jy} \mathrm{km} \mathrm{s}^{-1}$; $\mathrm{CH}_{3} \mathrm{OH}-\mathrm{E}\left(4_{+2,2}-3_{+1,2}\right): 4.8$, $8,11.2,14.4,17.6 \mathrm{Jy} \mathrm{km} \mathrm{s}^{-1} ; \mathrm{H}_{2} \mathrm{CO}\left(3_{2,2}-2,1\right): 3.24,5.4,7.56 \mathrm{Jy} \mathrm{km} \mathrm{s}^{-1}$; $\mathrm{H}_{2} \mathrm{~S}: 2.55,4.25,5.95,9.35 \mathrm{Jy} \mathrm{km} \mathrm{s}^{-1}$. The number below each molecular name is the $E_{\text {lower }}$ of the transition in units of $\mathrm{cm}^{-1}$.

\subsubsection{Compact Molecular Line Emission and Absorption}

As seen in Figure 8, emission from the higher excitation lines $\left(E_{l}>70 \mathrm{~cm}^{-1}\right)$ is concentrated toward SMA1. In several species, a secondary peak of emission appears toward SMA2. Indeed, the SMA2 peak in methyl formate and the lower lying transitions of methanol is almost equal to that of SMA1. A few vibrational states of $\mathrm{CH}_{3} \mathrm{OH}$ and $\mathrm{HC}_{3} \mathrm{~N}$ are detected exclusively toward SMA1, suggesting that it is warmer and/or denser than SMA2. Several species also show weak emission toward SMA4 and to the north and west of SMA6. Figure 9 shows the first moment maps for several representative species detected in emission, along with the $\mathrm{CN}$ molecule which is detected in absorption. Because of the possibility of confusion with outflowing or infalling gas, it is difficult to conclusively determine the systemic velocities of all of the $1.3 \mathrm{~mm}$ cores. Both SMA1 and SMA2 show enough consistency in a range of species with compact emission to estimate that $V_{l s r}($ SMA1 $)=-2.8 \pm 0.5 \mathrm{~km} \mathrm{~s}^{-1}$ and $V_{l s r}($ SMA2) $=$ $-4.5 \pm 0.5 \mathrm{~km} \mathrm{~s}^{-1}$. Compact emission from SMA4 is weak and shows a peak emission velocity of $V_{l s r}(\mathrm{SMA} 4)=-5 \pm 1$ $\mathrm{km} \mathrm{s}^{-1}$. No emission centered on SMA6 was detected.

The $\mathrm{CN}(2-1)$ lines are seen in absorption against most of the stronger compact continuum sources, while most of its extended emission is resolved out by the interferometer. None of the $1.3 \mathrm{~mm}$ continuum brightness temperatures $\left(T_{b}\right)$ are high enough at the current angular resolution to detect the $E_{l}=40 \mathrm{~cm}^{-1} \mathrm{H}_{2} \mathrm{CO}$ transition in absorption (negative) against it, though self-absorption is seen toward SMA1 and SMA2. Only SMA1 has a continuum $T_{b}(\sim 15 \mathrm{~K}$ at current resolu- tion) high enough to detect the $\mathrm{H}_{2} \mathrm{CO} E_{l}=7 \mathrm{~cm}^{-1}$ transition in (negative) absorption. Figure $10 \mathrm{a}$ and $\mathrm{b}$ show spectra of $\mathrm{HCOOCH}_{3}\left(E_{l}=77 \mathrm{~cm}^{-1}\right), \mathrm{H}_{2} \mathrm{CO}$ at $E_{l}=7$ and $40 \mathrm{~cm}^{-1}$, and $\mathrm{CN}\left(E_{l}=3.8 \mathrm{~cm}^{-1}\right)$ toward the SMA1 and SMA2 $1.3 \mathrm{~mm}$ continuum peaks. The absorption velocity of $\mathrm{CN}$ toward SMA1 is in good agreement with $V_{l s r}(\mathrm{SMA} 1)=-2.8 \pm 0.5 \mathrm{~km} \mathrm{~s}^{-1}$. The absorption seen in the $\mathrm{H}_{2} \mathrm{CO}\left(E_{l}=7 \mathrm{~cm}^{-1}\right)$ line is slightly redshifted by $\sim 0.5 \mathrm{~km} \mathrm{~s}^{-1}$ compared to $\mathrm{CN}$ and the $V_{l s r}$. Interestingly, the $E_{l}=40 \mathrm{~cm}^{-1} \mathrm{H}_{2} \mathrm{CO}$ line shows a self-absorption dip redshifted by $\sim 1 \mathrm{~km} \mathrm{~s}^{-1}$ relative to $\mathrm{CN}$ and the $V_{l s r}$ (see Fig. 10 a).

SMA2 shows $\mathrm{CN}$ absorption redshifted by $\sim 4 \mathrm{~km} \mathrm{~s}^{-1}$ from the $V_{l s r}(\mathrm{SMA} 2)=-4.5 \pm 0.5 \mathrm{~km} \mathrm{~s}^{-1}$, suggesting strong infall is present towards this source (see Fig. 10b). In contrast, the $E_{l}=40 \mathrm{~cm}^{-1} \mathrm{H}_{2} \mathrm{CO}$ emission peak is slightly blueshifted $\left(\sim 0.5 \mathrm{~km} \mathrm{~s}^{-1}\right)$ compared to other emission lines (see for example $\mathrm{HCOOCH}_{3}$ in Fig $10 \mathrm{p}$ ), while the $E_{l}=7 \mathrm{~cm}^{-1} \mathrm{H}_{2} \mathrm{CO}$ transition's peak is blueshifted by $2 \mathrm{~km} \mathrm{~s}^{-1}$. Neither $\mathrm{H}_{2} \mathrm{CO}$ line is Gaussian in shape, with the redshifted side of both transitions cutting off very sharply as might be expected from very strong, red-shifted self-absorption. Weak blueshifted $(-5$ to $-15 \mathrm{~km} \mathrm{~s}^{-1}$ ) $\mathrm{H}_{2} \mathrm{O}$ masers were also detected toward SMA2 so these masers and $\mathrm{H}_{2} \mathrm{CO}$ could be tracing a pole-on outflow, with the $V_{l s r}$ emission missing due to self-absorption and the redshifted $\mathrm{H}_{2} \mathrm{CO}$ outflow emission obscured by the continuum.

No compact molecular line emission is detected toward SMA3 so it is unclear if the $\mathrm{CN}$ absorption toward this core with a velocity of $\sim-2 \mathrm{~km} \mathrm{~s}^{-1}$ is also tracing infall or the $V_{l s r}$. Toward SMA4, CN shows absorption at $\sim-5 \mathrm{~km} \mathrm{~s}^{-1}$ in good agreement with the weak emission lines detected toward this source including $E_{l}=40 \mathrm{~cm}^{-1} \mathrm{H}_{2} \mathrm{CO}\left(V_{l s r}(\right.$ SMA4) $)=-5 \pm 1$ $\left.\mathrm{km} \mathrm{s}^{-1}\right)$. The $E_{l}=7 \mathrm{~cm}^{-1} \mathrm{H}_{2} \mathrm{CO}$ transition toward SMA4 is difficult to interpret as it is non-Gaussian in shape with a peak at $\sim-2.5 \mathrm{~km} \mathrm{~s}^{-1}$ and a significant blue-shifted shoulder. The $\mathrm{CN}$ absorption toward SMA6 has a velocity of $-4.0 \pm 0.5 \mathrm{~km} \mathrm{~s}^{-1}$, in good agreement with the velocity of a self-absorption dip seen in $E_{l}=7 \mathrm{~cm}^{-1} \mathrm{H}_{2} \mathrm{CO}$. Since no compact emission is detected towards this source it is unclear how this absorption velocity compares to the $V_{l s r}$ of SMA6.

Also of interest is the barely resolved SE-NW (or sometimes more E-W) velocity gradient across SMA1 evident in a number of compact species (see for example $\mathrm{HCOOCH}_{3}$ and $\mathrm{CH}_{3} \mathrm{OH}$ in Fig. 9]. The full width of the velocity gradient is about $\sim 5 \mathrm{~km} \mathrm{~s}^{-1}$ (from inspecting the line cubes), in reasonable agreement with the gradient inferred by Beuther et al. (2007) for $\mathrm{NH}_{3}(6,6)$ based on a double peaked profile. This gradient is discussed further in $\$ 4.5$.

\subsection{Mid-infrared Emission}

Figure 11 shows a three color mid-IR image of the I(N) region with methanol maser positions and integrated $\mathrm{SiO}$ and $1.3 \mathrm{~mm}$ continuum contours superposed. The extended 4.5 $\mu \mathrm{m}$ emission to the $\mathrm{SW}$ of the $\mathrm{I}(\mathrm{N})$ continuum has been reported previously by Hunter et al. (2006). In addition, a weak region of predominantly $4.5 \mu \mathrm{m}$ emission is also visible between the SMA6 continuum peak and the arc of $44 \mathrm{GHz}$ $\mathrm{CH}_{3} \mathrm{OH}$ masers $5^{\prime \prime}$ to the $\mathrm{SW}$; this region is coincident with the blueshifted side of the SMA6 outflow (Fig. 77). Clumpy knots of extended $4.5 \mu \mathrm{m}$ emission are also present to the NE of SMA1, and in the vicinity of SMA7. We also present for the first time, an unresolved source of $24 \mu \mathrm{m}$ emission located 

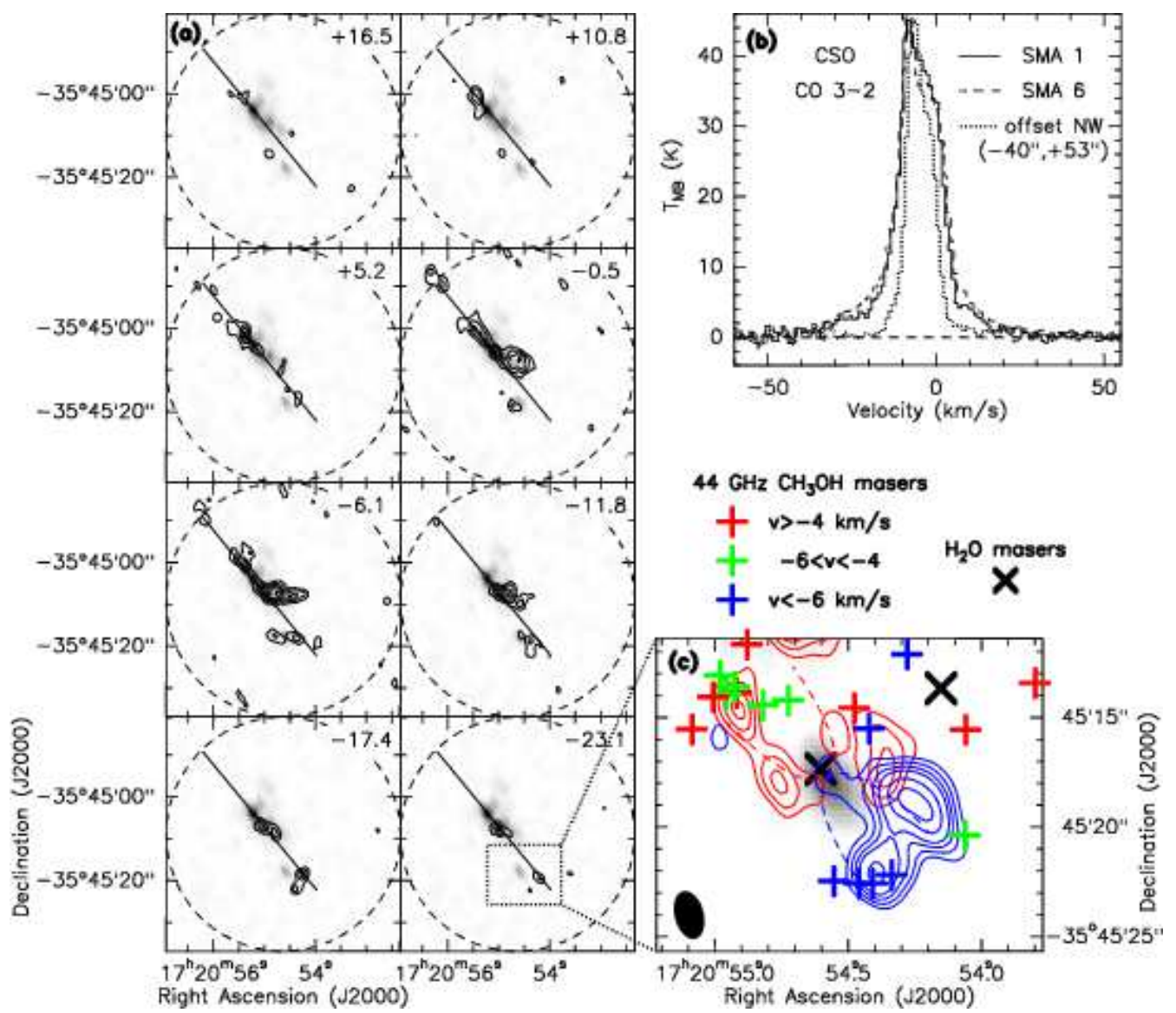

FIG. 7. - (a) Channel maps of $\mathrm{SiO} 5-4$. In each panel, the greyscale is the $1.3 \mathrm{~mm}$ continuum emission and the contours are $\mathrm{SiO}$ emission (levels $=0.29,0.48$, $0.67,0.86,1.05 \mathrm{Jy}$ ). The outflow axis is drawn at position angle $+39^{\circ}$. The dashed circle is the SMA primary beam. (b) CSO CO (3-2) spectra taken near SMA1, SMA6 and a position offset $-40^{\prime \prime},+53^{\prime \prime}$ to the northwest (away from any obvious outflow emission). The SMA1 spectrum was taken $2.9^{\prime \prime}$ from SMA1, and the SMA6 spectrum is the average of the three spectra taken closest to SMA6 $\left(5^{\prime \prime}, 7^{\prime \prime}\right.$, and $8^{\prime \prime}$ away). (c) Close up of SiO (5-4) outflow around SMA6. The contours show $\mathrm{SiO}(5-4)$ integrated emission, red contours show integrated intensity from +17 to $-6 \mathrm{~km} \mathrm{~s}^{-1}$ and blue contours show integrated intensity from from -8 to $-31 \mathrm{~km} \mathrm{~s}^{-1}$. The $\mathrm{CH}_{3} \mathrm{OH}$ and $\mathrm{H}_{2} \mathrm{O}$ maser positions are also shown. The solid black ellipse is the SMA synthesized beam.

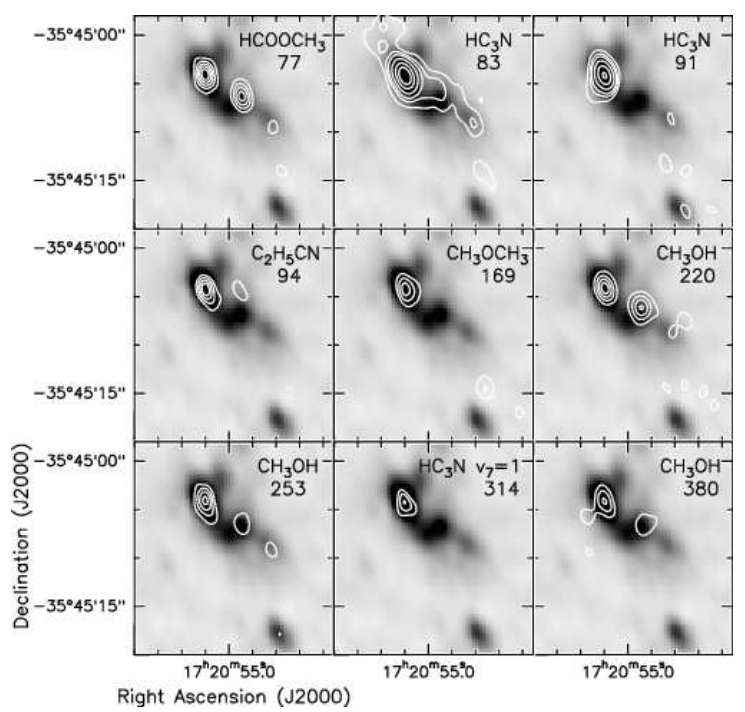

FIG. 8.- In each panel, the greyscale is the $1.3 \mathrm{~mm}$ continuum and the contours are drawn from moment-zero images of spectral lines. The contour levels are: $\mathrm{HCOOCH}_{3}: 2.1,4.2,6.3,8.4,10.5 \mathrm{Jy} \mathrm{km} \mathrm{s}^{-1} ; \mathrm{HC}_{3} \mathrm{~N}: 2.55,4.25$, $5.95,9.35,14.45,19.55 \mathrm{Jy} \mathrm{km} \mathrm{s}^{-1} ; \mathrm{C}_{2} \mathrm{H}_{5} \mathrm{CN}: 1.8,3.0,4.2,5.4 \mathrm{Jy} \mathrm{km} \mathrm{s}{ }^{-1}$; $\mathrm{CH}_{3} \mathrm{OCH}_{3}: 1.35,2.25,3.15 \mathrm{Jy} \mathrm{km} \mathrm{s}^{-1} ; \mathrm{CH}_{3} \mathrm{OH}(220): 1.89,3.78,5.67$, $7.56 \mathrm{Jy} \mathrm{km} \mathrm{s}{ }^{-1} ; 2.31,3.85,5.39,6.93 \mathrm{Jy} \mathrm{km} \mathrm{s}^{-1} ; \mathrm{HC}_{3} \mathrm{~N}\left(\mathrm{v}_{7}=1\right): 1.5,2.5$, $3.5 \mathrm{Jy} \mathrm{km} \mathrm{s}^{-1} ; \mathrm{CH}_{3} \mathrm{OH}(380): 2.1,3.5,4.9 \mathrm{Jy} \mathrm{km} \mathrm{s}^{-1}$. The numbers below each molecular name is the $E_{\text {lower }}$ of the transition in units of $\mathrm{cm}^{-1}$. $\sim 2^{\prime \prime}$ SW of SMA4. This figure demonstrates that with the exception of extended $4.5 \mu \mathrm{m}$ emission, and a single source of $24 \mu \mathrm{m}$ emission, NGC $6334 \mathrm{I}(\mathrm{N})$ is relatively dim in the mid-IR. Indeed, SMA4 appears to be the only millimeter continuum source directly associated with a mid-IR source. In contrast, the bright haze of red emission in the SW corner of Fig. 11 emanates from the periphery of the saturated midIR bright protocluster NGC 6334 I. The nature of the mid-IR emission is discussed in detail in $\S 4.1$ and $\S 4.2$.

\section{DISCUSSION}

\subsection{Presence of Multiple Outflows}

Due to the ubiquity of bipolar outflows from protostars, one would expect to find multiple outflows in a protostellar cluster. As discussed in Cyganowski et al. (2008) (and references therein), the $4.5 \mu \mathrm{m}$ IRAC passband can be dominated by molecular line emission from $\mathrm{H}_{2}$ and vibrationally-excited $\mathrm{CO}$ in regions of strong shocks such as those found in massive molecular outflows. To the NE of SMA1, the extended $4.5 \mu \mathrm{m}$ emission appears to be an extension of the large-scale NE-SW SMA1 outflow traced by $\mathrm{SiO}$ and other tracers of the extended emission (see Figs. 677 11). To the south where the outflows from SMA1 and SMA4 (westward flow) overlap, it is unclear which outflow the $4.5 \mu \mathrm{m}$ emission is arising from. Interestingly, the $4.5 \mu \mathrm{m}$ emission extends further than the $\mathrm{SiO}$ does to the west and SW. As described in $\$ 3.5$, 


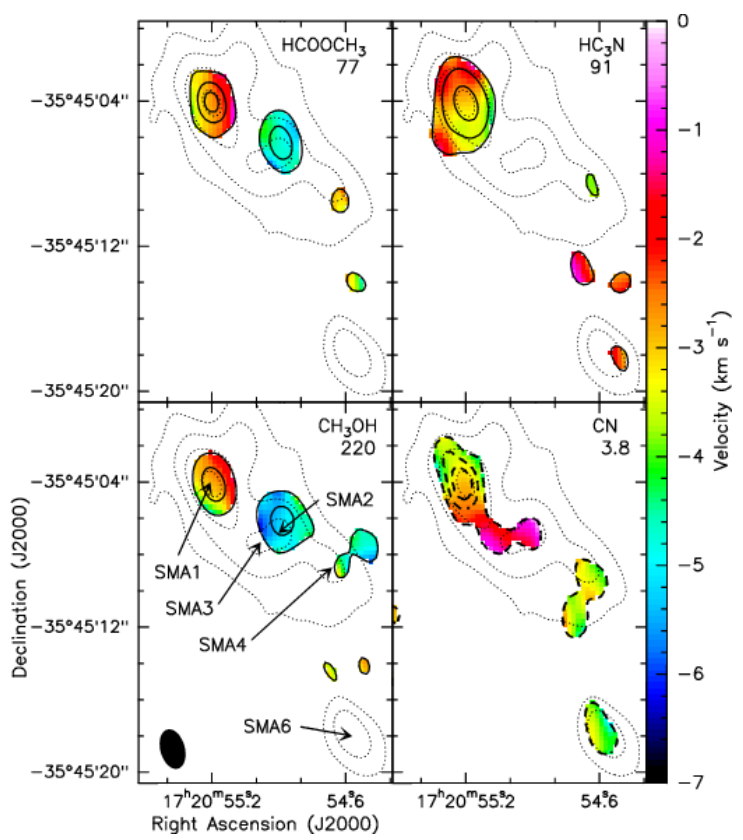

FIG. 9.- In each panel, the color scale shows the first moment map of the indicated molecular line, while the solid black contours depict the integrated intensity image of the line emission. In the case of $\mathrm{CN}$, the dashed contours indicate that the line appears in absorption. The levels are: $\mathrm{HCOOCH}_{3}=2.1$, 6.3, $10.5 \mathrm{Jy} \mathrm{km} \mathrm{s}^{-1} ; \mathrm{HC}_{3} \mathrm{~N}=2.55,5.95,14.45 \mathrm{Jy} \mathrm{km} \mathrm{s}{ }^{-1} ; \mathrm{CH}_{3} \mathrm{OH}=1.89$, $5.67 \mathrm{Jy} \mathrm{km} \mathrm{s}^{-1} ; \mathrm{CN}=-1.2,-2.4,-3.6 \mathrm{Jy} \mathrm{km} \mathrm{s}^{-1}$. In each panel, the thin dotted contours show the $1.3 \mathrm{~mm}$ continuum emission (levels $=60,180,390$, and $900 \mathrm{mJy}^{\text {beam }^{-1}}$ ). The numbers below each molecular name is the $E_{\text {lower }}$ of the transition in units of $\mathrm{cm}^{-1}$.
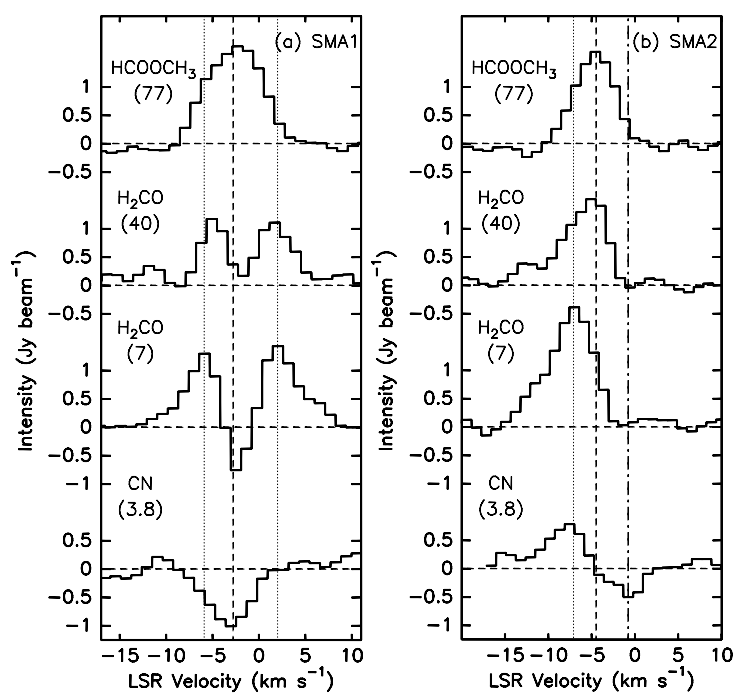

FIG. 10.- (a) Sample spectra toward the $1.3 \mathrm{~mm}$ continuum peak of SMA1. (b) Sample spectra toward the $1.3 \mathrm{~mm}$ continuum peak of SMA2. In both plots the dashed line indicates the $V_{l s r}$ and the numbers in parenthesis under the molecule names are the lower state energy in $\mathrm{cm}^{-1}$. In (a) the two dotted lines indicate the velocity of the peak red and blueshifted $\mathrm{H}_{2} \mathrm{CO}\left(E_{l}=7 \mathrm{~cm}^{-1}\right)$ emission. In (b) the dotted line indicates the velocity of the peak blueshifted $\mathrm{H}_{2} \mathrm{CO}\left(E_{l}=7 \mathrm{~cm}^{-1}\right)$ emission and the dot-dashed line indicates the velocity of maximum $\mathrm{CN}$ absorption.

SMA2 may harbor a more or less pole-on outflow as traced by blueshifted $\mathrm{H}_{2} \mathrm{O}$ masers and possibly $\mathrm{H}_{2} \mathrm{CO}$. As described in $\$ 3.6$ and $\$ 3.7$, a smaller scale NE-SW outflow also appears to emanate from SMA6, with blueshifted $\mathrm{SiO}$ and $44 \mathrm{GHz}$ $\mathrm{CH}_{3} \mathrm{OH}$ maser emission located to the south coincident with $4.5 \mu \mathrm{m}$ emission (Figs. 77. 11 .
The ATCA molecular line data reported by Beuther et al. (2007, 2008) (HCN(1-0) and $\mathrm{NH}_{3}$ for example) show good overall morphological agreement with the largescale emission emanating from SMA1 observed with the SMA (see for example Figs. 67/11). In particular, the complex kinematics of the extended emission were also noted by Beuther et al. and ascribed to possible precession. This idea is in good agreement with the discovery of a possible tight binary in SMA1b and SMA1d (separated by $\gtrsim 800 \mathrm{AU}$ ) if these two sources do in fact represent two different protostars (see for example Chandler et al. 2005; Anglada et al. 2007; Cunningham et al. 2009). Although Figs. 677,b,c 11 show $\mathrm{SiO}$ and other molecules in the vicinity of SMA6, Beuther et al. (2007, 2008) did not detect any significant molecular emission in this region. However, SMA6 was near the edge of the ATCA primary beam.

It is notable that the orientation of the NE-SW outflow inferred to originate from SMA1 is different from the largescale SE-NW outflow direction inferred from single-dish $\mathrm{SiO}(5-4)$ data with $23^{\prime \prime}$ resolution presented by Megeath \& Tieftrunk (1999). In their single dish data, blueshifted emission is located to the SE of $\mathrm{I}(\mathrm{N})$ in the vicinity of SM2 (Fig. 4.3), while blueshifted and redshifted emission overlap in the vicinity of $\mathrm{I}(\mathrm{N})$. Toward $\mathrm{I}(\mathrm{N})$, the $\mathrm{P}-\mathrm{V}$ diagram presented by Megeath \& Tieftrunk (1999) shows good agreement with the velocity range observed in the SMA $\mathrm{SiO}(5-4)$ data $(-30$ to $+17 \mathrm{~km} \mathrm{~s}^{-1}$, Fig. 77,b), and indeed the SEST data did not have sufficient spatial resolution to resolve the SMA sizescale flow from SMA1. Moreover, Megeath \& Tieftrunk (1999) also observe a clump of shocked $2.2 \mu \mathrm{m} \mathrm{H}_{2}$ emission coincident with the northeastern lobe of the SMA1 outflow and extended $4.5 \mu \mathrm{m}$ emission. Thus, it seems likely that the single outflow reported by Megeath \& Tieftrunk (1999), is in fact at least two outflows - one consistent with the NE-SW flow detected by the SMA and a second in the vicinity of SM2 and the C1 and $\mathrm{C} 2$ water masers. This second outflow would also explain the widespread $44 \mathrm{GHz} \mathrm{CH} \mathrm{CH}_{3} \mathrm{OH}$ maser emission observed in this region. While this seems the most probable interpretation of the data, it is also possible that spatial filtering of the SMA data on sizescales $\gtrsim 20^{\prime \prime}$ have played a role in the apparent discrepancy. Unfortunately, the SM2 area is beyond the primary beam of the SMA observations, so additional data will be required to explore this region of activity further.

\subsection{Nature of Mid-infrared Emission}

To assess the nature of the mid-IR emission in more detail we analyzed the 1-8 $\mu \mathrm{m}$ flux densities of several knots in the vicinity of $\mathrm{I}(\mathrm{N})$ that morphologically appear to be dominated by shock line emission. We included data from the Spitzer IRAC bands (see for example Ybarra \& Lada 2009), but also near-infrared $J, H$, and $K_{s}$ fluxes (see for example Smith 1995). To derive the physical conditions, we have implemented a set of shock models similar to that of Ybarra \& Lada (2009) (see also Neufeld \& Yuan 2008; Smith \& Rosen 2005; Smith et al. 2006). We calculate the equilibrium excitation of molecular hydrogen $\left(\mathrm{H}_{2}\right)$ in a grid of temperatures $(1000$ $5000 \mathrm{~K})$ and densities $\left(10^{2}-10^{6} \mathrm{~cm}^{-3}\right)$ using the escape probability code Radex van der Tak et al. 2007, since the optical depths are low, the escape probability formalism is not critical, but the code was still very useful to read the extensive tables of molecular data). We use excitation rates from collisions with $\mathrm{H}_{2}$ and $\mathrm{He}$ from Le Bourlot et al. (1999, 2002) including reactive collisions as prescribed therein, and from 


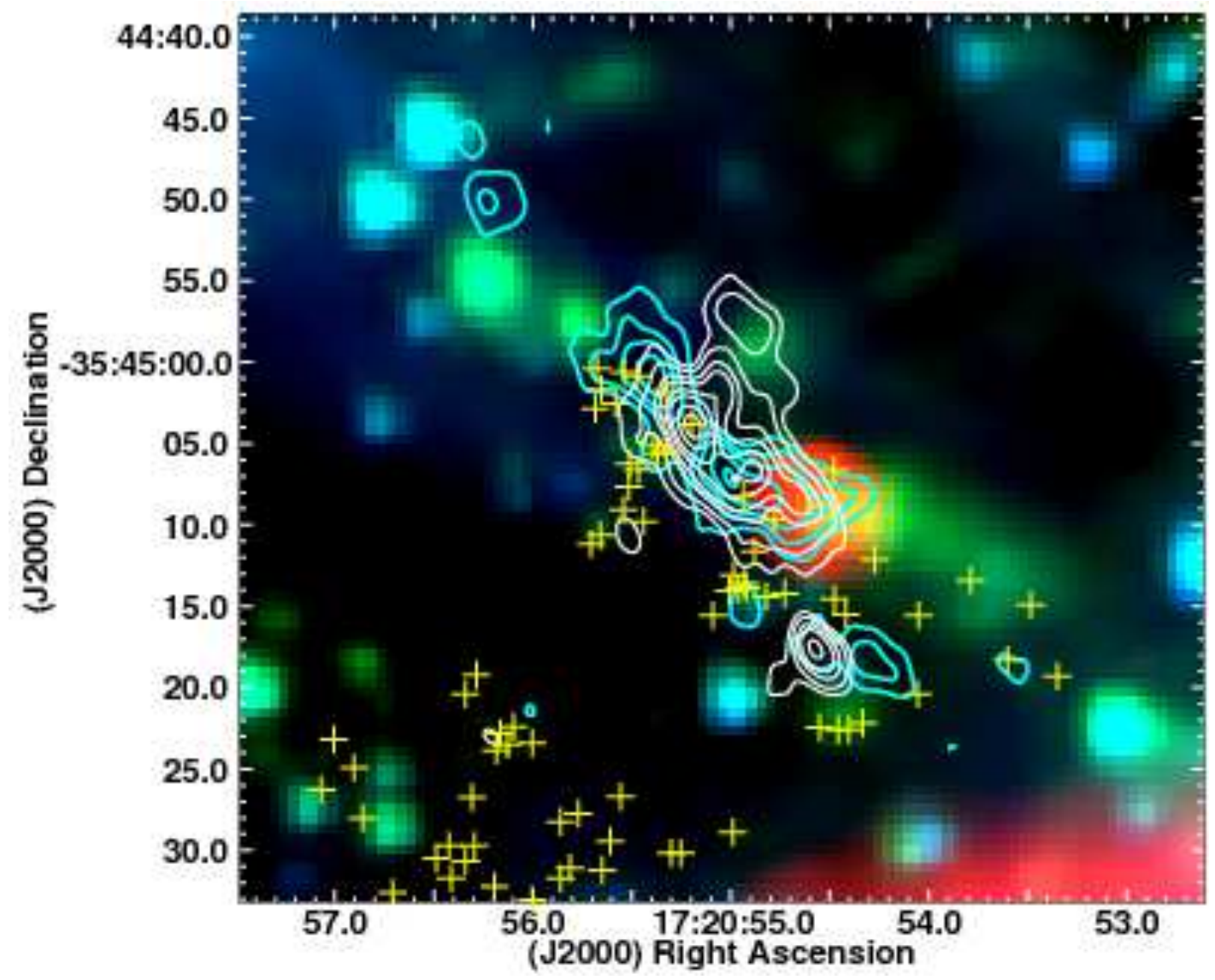

FIG. 11.- Spitzer 3-color image with RGB mapped to 24, 4.5, and $3.6 \mu \mathrm{m}$. White $1.3 \mathrm{~mm}$ continuum and cyan SiO integrated intensity contours are overlaid, along with yellow + symbols showing the locations of the $44 \mathrm{GHz} \mathrm{CH}_{3} \mathrm{OH}$ masers. The $1.3 \mathrm{~mm}$ continuum contour levels are the same as Fig. 1, and the SiO (5-4) integrated emission contour levels are 6,10,14, 20, $26 \mathrm{mJy}_{\text {beam }}{ }^{-1} * \mathrm{~km} \mathrm{~s}^{-1}$.

collisions with $\mathrm{H}$ from Wrathmall et al. (2007). Quadrupole transition probabilities are from Wolniewicz et al. (1998). We convolve the line intensities with Spitzer (Reach et al. 2005) and $2 \mathrm{MASS}^{12}$ filter transmission profiles to calculate bandaverage fluxes as specified in appendix A of Robitaille et al. (2007).

The analysis of the shocked emission is complicated by diffuse PAH emission, especially in the 5.8 and $8.0 \mu \mathrm{m}$ images. We performed several different background subtractions to attempt to isolate the $\mathrm{H}_{2}$ emission, with the assumption that most of the emission at $4.5 \mu \mathrm{m}$ is $\mathrm{H}_{2}$ (there are no PAH emission features in that band). Naturally this decomposition is subject to uncertainty, so the precision of shock physical parameters is limted by this observational fact. We find that the near and mid-IR broadband flux ratios are most consistent with the hottest post-shock temperatures $(\sim 4000 \mathrm{~K})$, indicating that these are strong shocks as would be expected from a powerful massive stellar outflow. The density in the shock cooling zone is harder to constrain because density changes the broadband flux ratios less than does temperature (see Fig. 2 in Ybarra \& Lada 2009), and because foreground extinction can mimic a decrease in emitting material density. The fluxes here are broadly consistent with densities of $10^{3}-10^{5} \mathrm{~cm}^{-3}$. Once we have constrained the physical parameters in the $\mathrm{H}_{2}$ emitting zone, we can translate mid-infrared fluxes into total shock luminosity. For shock recombination zones $(\mathrm{T} \simeq 4000 \mathrm{~K})$ our models indicate that
$6 \%$ of the total $\mathrm{H}_{2}$ line emission comes out in the IRAC $4.5 \mu \mathrm{m}$ band. The NGC6334I(N) outflow emits $15 \pm 3$ Jy over $100 \pm 20$ square arcseconds (sum of both lobes). At a distance of $1.7 \mathrm{kpc}$, that corresponds to $2300 \pm 300 \mathrm{~L}_{\odot}$ total luminosity in shocked molecular hydrogen, with an average column density of $3.5 \pm 0.5 \times 10^{21} \mathrm{~cm}^{-2}$, for a total of $0.1 \mathrm{M}_{\odot}$ of hot shocked material (that which is emitting at the highest temperatures - a much larger mass of cooler and entrained material is present in the outflow as well, simply not emitting in the near infrared).

SMA4 is the only source in $\mathrm{I}(\mathrm{N})$ associated with $6.7 \mathrm{GHz}$ $\mathrm{CH}_{3} \mathrm{OH}$ masers (see $\$ 3.3 .2$ ), and in $\$ 3.6$ we report the detection of a $24 \mu \mathrm{m}$ source $2^{\prime \prime}$ to the $\mathrm{SW}$ of the $1.3 \mathrm{~mm}$ continuum peak. Class II $6.7 \mathrm{GHz} \mathrm{CH}_{3} \mathrm{OH}$ masers are thought to be radiatively pumped by warm dust, and are found almost exclusively in regions of massive star formation (Cragg et al. 2002, 2005; Minier et al. 2003), providing strong evidence that SMA4 is a massive protostar. Two scenarios exist for the nature of the $24 \mu \mathrm{m}$ emission: (1) it may trace emission from hot dust near a central protostar and is the $24 \mu \mathrm{m}$ counterpart to SMA4 or (2) it traces emission from hot dust in the walls of the cavity formed by the outflow traced by extended $4.5 \mu \mathrm{m}$ and molecular line emission, as well as 44 $\mathrm{GHz}$ methanol masers W/SW of SMA4. Recent work by De Buizer et al. (2005); De Buizer (2007) favor the latter interpretation. Higher-resolution mid-infrared data would help to distinguish between these possibilities for SMA4. The $24 \mu \mathrm{m}$ flux is $0.45 \pm 0.04 \mathrm{Jy}$, or $2 L_{\odot}$ (just in the $24 \mu \mathrm{m}$ band).

${ }^{12}$ http://www.ipac.caltech.edu/2mass/releases/allsky/do\$rovstarbenmitrmughly $5 \%$ of their luminosity in the $24 \mu \mathrm{m}$ 
band over a relatively large range of evolutionary state, so if this source represents a significant fraction of the luminosity of the driving protostar, the latter would have an approximate luminosity of only about $40 L_{\odot}$. This low luminosity lends support to the interpretation that the $24 \mu \mathrm{m}$ emission is coming from hot dust away from the central source, such as on an outflow cavity, and that the driving source itself is still highly embedded and extinguished even at $24 \mu \mathrm{m}$.

\subsection{What are the $44 \mathrm{GHz}$ Methanol Masers Tracing?}

The $44 \mathrm{GHz}$ Class I $\mathrm{CH}_{3} \mathrm{OH}$ maser line is often found in regions of high-mass star formation (Haschick et al. 1990; Slysh et al. 1994; Kurtz et al. 2004; Val'Tts \& Larionov 2007; Pratap et al. 2008). Like other Class I masers (Plambeck \& Menten 1990), it is often found separated from compact HII regions and in many cases appears to be associated with shocked gas created by bipolar outflows (e.g. Kurtz et al. 2004; Sandell et al. 2005). While this maser has been found within other millimeter protoclusters such as S255N and G31.41+0.31 (Cyganowski et al. 2007; Araya et al. 2008), the number and spatial distribution of maser spots is particularly large in NGC $6334 \mathrm{I}(\mathrm{N})$. In all of these studies including the current one, the $44 \mathrm{GHz} \mathrm{CH} 3 \mathrm{OH}$ masers tend to lie within a few $\mathrm{km} \mathrm{s}^{-1}$ of the systemic velocity even though they typically trace powerful outflows. This dichotomy is likely due to the fact that an enhanced column of $\mathrm{CH}_{3} \mathrm{OH}$ gas with sufficient velocity coherence along the lineof-sight is most likely to be found where a shock is moving perpendicular to the line-of-sight (i.e. a shock viewed edgeon). The same effect is believed to give rise to the narrow $\mathrm{NH}_{3}$ $(3,3)$ maser emission located near the ends of the protostellar jet from IRAS 20126+4104 (Zhang et al. 1999).

As described in $\$ 4.1$, it is possible that the $44 \mathrm{GHz}$ masers located in the SE portion of the observed field of view are associated with an outflow originating from the relatively unexplored region of continuum emission SM2. The discovery of an X-ray source near SM2 indicates that additional cluster members may be present in this area. A more general conclusion can be drawn from the relatively widespread extent $\left(45^{\prime \prime} \sim 1.2 \times 10^{18} \mathrm{~cm}\right)$ of $44 \mathrm{GHz}$ maser emission, an extent that is shared by ammonia $(1,1)$ and $(2,2)$ line and $450 \mu \mathrm{m}$ continuum emission (see Fig. ). For a single dominant YSO to maintain this region at a conservative minimum temperature of $30 \mathrm{~K}$ (the ammonia temperature from Kuiper et al. (1995) and average dust temperature from Sandell (2000)) would require a central luminosity of $5 \times 10^{5} \mathrm{~L}_{\odot}$ (e.g. Scoville \& Kwan 1976) which is 30 times higher than the value $\left(1.7 \times 10^{4} \mathrm{~L}_{\odot}\right)$ inferred from the farinfrared spectral energy distribution for this region (Sandell 2000). Thus, these phenomena must be driven by the collective activity of a number of cluster members.

\subsection{Temperatures of the Hot Core Sources SMA1 and SMA2}

When several transitions of the same molecule spanning a range of energies are observed, temperatures can be inferred from the rotation diagram method (see for example Goldsmith \& Langer 1999), using the relations

$$
\frac{N_{u}}{g_{u}}=\frac{3 k}{8 \pi^{3} \nu g_{I} g_{K}} \frac{1}{\mu^{2} S} \int S_{\nu} d \nu,
$$

and

$$
\log \left(N_{u} / g_{u}\right)=\log \left(N_{\text {tot }} / Q\left(T_{\text {rot }}\right)\right)-0.4343 E_{u} / k T_{\text {rot }},
$$

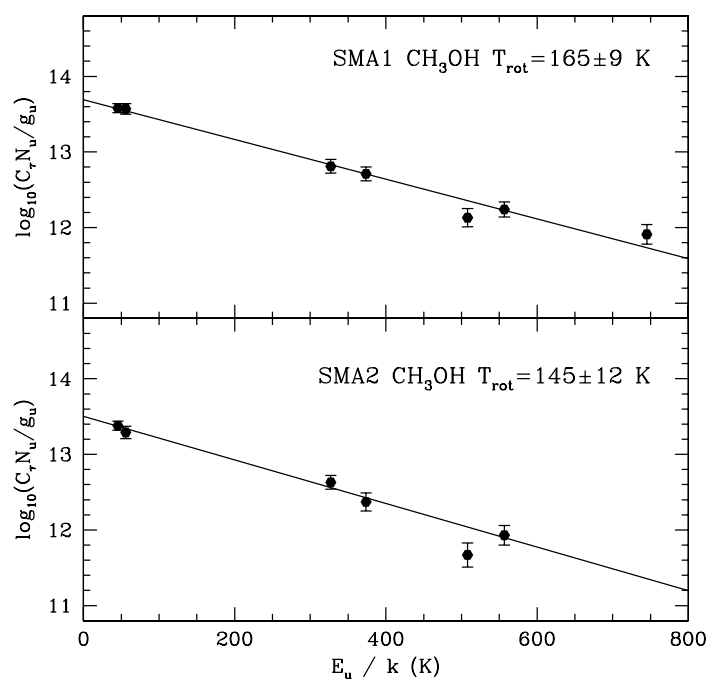

FIG. 12.- Rotation diagrams for the $\mathrm{CH}_{3} \mathrm{OH}$ transitions observed for SMA1 (top) and SMA2 (bottom); the fitted temperatures are indicated. The column densities have been corrected for optical depth effects as described in $\S 4.3$.

where $\mathrm{k}$ is Boltzmann's constant, $\nu$ is the frequency, $g_{I}$ and $g_{K}$ are the degeneracies associated with the nuclear spin and $\mathrm{k}$ quantum number respectively, $\mu^{2}$ is the square of the dipole matrix element, $S$ is the statistical line strength, $\int S_{\nu} d v$ is the observed integrated intensity of the transition, $N_{\text {tot }}$ is the total column density, $Q\left(T_{\text {rot }}\right)$ is the partition function evaluated at the rotation temperature $T_{r o t}$, and $E_{u}$ is the upper state energy of the transition. There are seven transitions of $\mathrm{CH}_{3} \mathrm{OH}$ detected toward SMA1 and six toward SMA2 (including both A and $E$ type transitions) that can be used for this purpose. The $\int S_{\nu} d v$ for each transition was calculated from Gaussian fits to the line emission at the $1.3 \mathrm{~mm}$ continuum peaks. The rotation temperature resulting from a weighted least squares fit to the data is $189 \pm 19 \mathrm{~K}$ for SMA1 and $154 \pm 14 \mathrm{~K}$ for SMA2.

However, as demonstrated by Goldsmith \& Langer (1999) and others, line optical depth can artificially increase the derived rotation temperature if not corrected for. In general the line optical depth in LTE can be calculated from

$$
\tau=\frac{8 \pi^{3}}{3 h} \frac{N_{o}}{g_{o}} \mu^{2} S e^{-E_{u} / k T_{e x}}\left(e^{h \nu / k T_{\text {rot }}}-1\right) \frac{1}{\Delta v},
$$

where $h$ is Planck's constant, $N_{o} / g_{o}$ is the column density of the ground state divided by the ground state degeneracy, $T_{e x}$ is the excitation temperature, and $\Delta v$ is the FWHM line width. Then the left hand side of Eq. 2 is modified to $\log \left(C_{\tau} N_{u} / g_{u}\right)$ where $C_{\tau}=\tau /\left(1-e^{-\tau}\right)$. Since we do not know the ground state column density, this equation cannot be used directly to calculate $\tau$ for each transition. Unfortunately, in the $\mathrm{I}(\mathrm{N})$ SMA dataset we do not have a suitable isotopologue that can be used to infer the $\mathrm{CH}_{3} \mathrm{OH}$ optical depth either (see for example Brogan et al.2007). Instead, we have iteratively solved for the optical depth and $T_{r o t}$ that produces the best $\chi^{2}$ fit to the data (i.e. minimizes the scatter). Using this technique we find the best fit occurs for a $T_{r o t}=165 \pm 9 \mathrm{~K}$ for SMA1 and $T_{r o t}=145 \pm 12 \mathrm{~K}$ for SMA2 (see Figure $12 \mathrm{k}$ ), and modest optical depths of 1.75 for SMA1 and 0.5 for SMA2 for the observed transition that would have the highest optical depth $\left(\mathrm{CH}_{3} \mathrm{OH}-\mathrm{E}\left(4_{+2,2}-3_{+1,2}\right)\right)$ at $\left.218.4400 \mathrm{GHz}\right)$. While not dramatically different from the $T_{\text {rot }}$ derived without opti- 
cal depth correction, these corrected temperatures are clearly more accurate. We do not have enough transitions to do a more sophisticated non-LTE analysis or to compare the results for $A$ and $E$ transitions independently (see for example Sutton et al. 2004). However, we note that for the $\mathrm{CH}_{3} \mathrm{OH}-\mathrm{E}$ $\left(5_{+1,4}-4_{+2,2}\right)$ transition (at $216.9456 \mathrm{GHz}$ ) which we have in common with the Sutton et al. (2004) methanol survey of the hot core source $\mathrm{W} 3(\mathrm{OH})$ with comparable spatial resolution, the derived opacity is similar for the two sources $(0.45$ for SMA1 in $\mathrm{I}(\mathrm{N})$ compared to 0.65 for $\mathrm{W} 3(\mathrm{OH}))$. Indeed the derived temperatures for SMA1 and SMA2 are also very similar to that of W3(OH) $\left(T_{\text {rot }}=140 \pm 10 \mathrm{~K}\right.$, Sutton et al. 2004).

The optical depth implied by the brightness temperature of the strongest methanol transition $\left(4_{+2,2}-3_{+1,2}, 24 \mathrm{~K}\right)$ toward SMA1 is only 0.16 assuming that $T_{k i n}=T_{r o t}=165 \mathrm{~K}$ and the emitting region is the size of the LSB synthesized beam $(2$ !' $39 \times 1$ !' 55$)$. An optical depth of 1.75 for this transition implies that the emitting region is actually only $0 . " 8$ in size (1400 AU). This size is in good agreement with the fitted size $\left(0 . \prime 9 \times 0 . \prime 7, \mathrm{PA}=162^{\circ}\right)$ of the integrated intensity measured from the compact, but still strong $\mathrm{CH}_{3} \mathrm{OH}-\mathrm{A}^{+}\left(16_{1,16}-15_{2,13}\right)$ transition $\left(E_{l}=219.8 \mathrm{~cm}^{-1}\right)$ at $227.8147 \mathrm{GHz}$. This size implies that the hot core region encompasses both SMA1b and SMA1d (see Fig. 2b).

The column densities of $\mathrm{CH}_{3} \mathrm{OH}$ for SMA1 and SMA2 are $(1.0 \pm 0.4) \times 10^{17} \mathrm{~cm}^{-2}$ and $(5.6 \pm 0.6) \times 10^{16} \mathrm{~cm}^{-2}$, respectively, an order of magnitude less than Sutton et al. (2004) find for $\mathrm{W} 3(\mathrm{OH})$. Sutton et al. (2004) find a $\mathrm{CH}_{3} \mathrm{OH}$ abundance of $\sim 2 \times 10^{-6}$ for $\mathrm{W} 3(\mathrm{OH})$. Using single dish observations of infrared dark clouds (IRDCs), Leurini et al. (2007) find abundances an order of magnitude smaller for the IRDC cores. Using this range of abundance and assuming that the hot core size is $1400 \mathrm{AU}$, we estimate that the $\mathrm{H}_{2}$ column density is $(0.5-5) \times 10^{23} \mathrm{~cm}^{-2}$ and the $\mathrm{H}_{2}$ density is $(0.2-2) \times 10^{7} \mathrm{~cm}^{-3}$ for SMA1 and about a factor of two smaller for SMA2.

Using the ATCA, Beuther et al. (2008) observed $\mathrm{CH}_{3} \mathrm{CN}\left(5_{K}-4_{K}\right)$ up to $\mathrm{K}=4$ toward SMA1 and SMA2 and derived a rotation temperature of $T_{\text {rot }}=170 \pm 50 \mathrm{~K}$ for SMA2 in good agreement with our $\mathrm{CH}+3 \mathrm{OH}$ measurement $(145 \pm 12 \mathrm{~K})$. The optical depth in SMA1 was too high to obtain a $\mathrm{CH}_{3} \mathrm{CN} T_{\text {rot }}$ for SMA1 in good agreement with our finding that this source has a higher column density than SMA2. Also using the ATCA, Beuther et al. (2007) detected $\mathrm{NH}_{3}(5,5)$, and $(6,6)$ emission toward both SMA1 and SMA2 confirming that $T_{k}$ is greater than $100 \mathrm{~K}$, though it was not possible to derive more accurate estimates from those data.

\subsection{The Nature of the $1.3 \mathrm{~mm}$ Sources \\ 4.5.1. Spectral Energy Distributions}

Because no higher frequency arcsecond-resolution data exist on this region, the determination of the nature of the $1.3 \mathrm{~mm}$ sources hinges entirely upon accurate measurements of their spectral index toward longer wavelengths (thus motivating our careful re-reduction of the available data). The comparatively poor angular resolution of the current 1.3 and $3.4 \mathrm{~mm}$ data compared to the $7 \mathrm{~mm}, 1.3 \mathrm{~cm}$, and $3.6 \mathrm{~cm}$ data makes it challenging to disentangle the free-free versus dust contributions to these sources. Figure 13 shows the spectral energy distributions (SEDs) of SMA1, SMA4, and SMA6 based on the flux densities provided in Table 1 The different resolutions of the different datasets are definitely a concern. However, in many cases the emission does not appear to be resolved even in the highest resolution observations of SMA4, and to some degree SMA6. In that case, as long as no significant emission is resolved out, the data will still be comparable despite the differing resolutions. Caveats for a few sources are described in detail below.

For SMA1, which contains at least four components at the longer wavelengths, we show both the integrated emission over the whole SMA1 region, and SMA1b by itself at wavelengths longer than $7 \mathrm{~mm}$ for comparison. The integrated emission from SMA1 shows evidence for both a free-free and an optically thin dust component. The integrated dust spectral index of $\alpha_{\text {dust }}=3.8 \pm 0.1\left(S_{\nu} \propto \nu^{\alpha}\right)$ was determined by a linear least-squares fit to the three flux densities from $7 \mathrm{~mm}$ to $1.3 \mathrm{~mm}$ (for SMA1 at $7 \mathrm{~mm}$ this is the sum of emission from SMA1a,b,c, and d; the free-free contribution is an order of magnitude smaller and hence negligible in the dust fit). On smaller scales, we also find that the $7 \mathrm{~mm}$ emission from SMA1b is dominated by dust, while the $3.6 \mathrm{~cm}$ emission is consistent with optically-thin free-free emission with a spectral index of $\nu^{-0.1}$, such as that from an H II region with an electron density of $3 \times 10^{4} \mathrm{~cm}^{-3}$, temperature of $10000 \mathrm{~K}$ and a diameter of $0.3^{\prime \prime}$ (510 AU). At the intermediate wavelength of $1.3 \mathrm{~cm}$, these two emission mechanisms are comparable for SMA1b, with $\approx 30 \%$ of the emission arising from dust. This is a notable result since $1.3 \mathrm{~cm}$ data are typically assumed to be free of dust.

The emission from SMA1a may all be due to dust since it is not detected longward of $7 \mathrm{~mm}$; the $3 \sigma 1.3 \mathrm{~cm}$ upper limit of $\sim 0.18 \mathrm{mJy}$ implies that the spectral index must be $>2.4$. In contrast, both SMA1c and SMA1d are detected at $1.3 \mathrm{~cm}$ and have a $1.3 \mathrm{~cm}$ to $7 \mathrm{~mm}$ spectral index of $\operatorname{sim} 2$. This spectral index could be due to optically thick dust emission which seems very unlikely at these wavelengths, optically thick freefree emission such as one might observe around a very dense hypercompact $\mathrm{H}$ II region, or a combination of optically thin free-free and dust emission. If we take the total observed flux density of SMA1 at $1.3 \mathrm{~cm}(1.07 \mathrm{mJy}$, i.e. the sum of SMA1a upper limit and SMA1b,c,and d detections) and subtract the total dust model $(0.50 \mathrm{mJy})$ and the SMA1b free-free emission model $(0.35 \mathrm{mJy})$, we find that $0.22 \mathrm{mJy}$ remains, suggesting that the integrated dust model cannot account for all of the SMA1c+SMA1d emission. Thus, one or both of SMA1c and SMA1d must have a weak free-free component, and since these objects have $1.3 \mathrm{~cm}$ flux densities of 0.27 and $0.30 \mathrm{mJy}$, respectively (greater than the residual), both must also have a dust component. If all of the free-free residual $(0.22 \mathrm{mJy})$ belonged to either SMA1c or SMA1d, the $3 \sigma 3.6 \mathrm{~cm}$ upper limit of $\sim 0.12 \mathrm{mJy}$ implies a free-free spectral index $>0.6$, consistent with a thermal jet, stellar wind, or hypercompact $\mathrm{H}$ II region. If instead the residual free-free emission is about equally split between SMA1c and SMA1d both could harbor small optically thin H II regions in addition to a dust component.

Understanding the nature of SMA4 is complicated by the lack of positional agreement between the $1.3-3.4 \mathrm{~mm}$ data compared to the longer wavelength data $(3.6 \mathrm{~cm}, 1.3 \mathrm{~cm}$, and

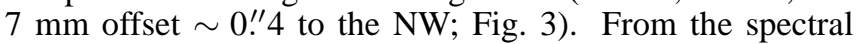
break in the SMA4 SED around $7 \mathrm{~mm}$ shown in Fig. 13 it is clear that both dust and free-free emission is present in this region. The spectral index of the SMA4 emission between 1.3 and $3.4 \mathrm{~mm}$ is $\alpha_{\text {dust }}=4.4 \pm 0.3$, while the spectral index between $3.6 \mathrm{~cm}$ and $7 \mathrm{~mm}$ is $\alpha_{f f}=0.63 \pm 0.25$ suggesting either a hypercompact $\mathrm{H}$ II region with a turnover around $7 \mathrm{~mm}$ (see for example the review by Lizano 2008), or a 

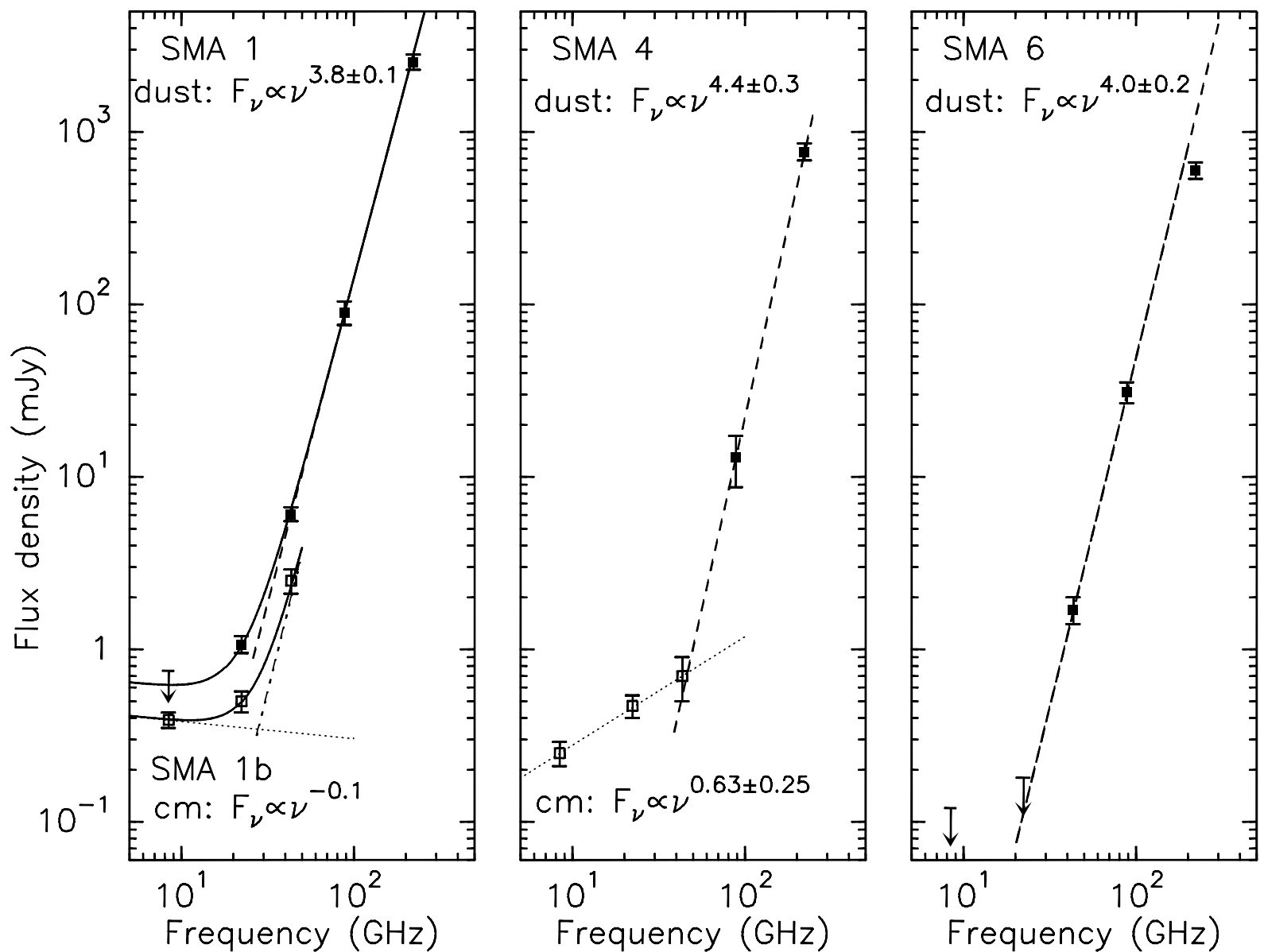

FIG. 13. - Spectral energy distributions for sources SMA1, SMA4, and SMA6. For SMA1, the sum of the emission of SMA1a,b,c, and d are shown as solid squares, while the flux density as a function of frequency for SMA1b is shown as open squares for $\nu<43 \mathrm{GHz}$. Open squares are also used for the $\nu<43 \mathrm{GHz}$ emission for SMA4 to indicate that they may not be from the same source as the higher frequency data, the fits in this case assume case 3 described in S4.5.1. Emission models from dust are shown as dashed (or dot-dash in the case of SMA1b) lines, while emission models for free-free emission are shown as dotted lines. For SMA1 the sum of the dust and free-free emission models, described in detail in §4.4.1, is shown as a solid line.

thermal jet/stellar wind (Reynolds 1986). Overall, the data are consistent with the following three possibilities: (1) the emission all arises from the same source which contains both dust and a hypercompact $\mathrm{H}$ II region (i.e. the position offsets are just within the combined position uncertainties); (2) Two sources are present: one is a compact dust source with no detectable free-free emission (1.3 - $3.4 \mathrm{~mm}$ emission) and the other (7 $\mathrm{mm}-3.6 \mathrm{~cm}$ emission) is a hypercompact H II region ( $\gtrsim 700 \mathrm{AU}$ away); or (3) the free-free emission arises from a one-sided thermal jet driven by the dust source of case (2). If we assume case (1) and fit the centimeter wavelength emission from SMA4 after extrapolating and subtracting the dust spectrum, we find that a free-free component with a constant density, temperature, and size of $1.2 \times 10^{6} \mathrm{~cm}^{-3}, 8000 \mathrm{~K}$, and $53 \mathrm{AU}$ best approximates the 3.6 and $1.3 \mathrm{~cm}$ data points (see Hunter et al. 2008, for the details of the free-free emission model). However, this model overpredicts the $7 \mathrm{~mm}$ flux density by a significant margin. More sophisticated freefree emission models, such as those with a density gradient and/or a shell geometry, may provide a better match to the $7 \mathrm{~mm}$ data (see for example Avalos et al. 2006). However, the present data are insufficient in angular resolution and number of spectral measurements to meaningfully constrain a more detailed model. For case (2), it is even more difficult to fit the $7 \mathrm{~mm}$ data with a hypercompact $\mathrm{H}$ II region model because the spectrum from $3.6 \mathrm{~cm}$ to $7 \mathrm{~mm}$ is so linear, and yet not steep enough to still be optically thick (i.e. spectral index of 2). Thus, case (3) seems to best fit the current data (see Fig. 13. with the $3.6 \mathrm{~cm}$ to $7 \mathrm{~mm}$ data having a typical jet or stellar wind spectral index ( $\alpha_{f f}=0.6$ Reynolds 1986), and no dust component. We note that in the jet interpretation for the $\mathrm{cm}-\lambda$ emission (case 3 ) the $\sim 0 . \prime 4$ offset of the $\mathrm{cm}-\lambda$ to the west is in good agreement with the fact that a westward outflow appears to emanate from SMA4.

Although there is an offset $\sim 0 ! 3$ to the north between the $7 \mathrm{~mm}$ and $1.3 \mathrm{~mm}$ emission toward SMA6, the fact that the shape of the continuum contours at these two wavelengths is similar, and most of the apparent offset is along the long axis of the $1.3 \mathrm{~mm}$ beam makes it quite plausible that they are in fact coincident (also note that the offset is not in the same direction as that of SMA4). Indeed, the emission from SMA6 can be explained completely by optically-thin dust emission (at the current level of sensitivity). The dust spectral index from a linear fit to the three flux densities from $7 \mathrm{~mm}$ to $1.3 \mathrm{~mm}$ is $\alpha=4.0 \pm 0.2$. If we assume all the $1.3 \mathrm{~mm}$ emission arises from a region with the size measured from the higher resolution $7 \mathrm{~mm}$ image, $\left(0 .{ }^{\prime \prime} 9 \times 0 .{ }^{\prime \prime} 7\right)$, the brightness temperature is $T_{b}=25 \mathrm{~K}$ setting a lower limit on the dust temperature. There is a hint of a break towards a flatter spectral index at $1.3 \mathrm{~mm}$ for SMA6, as would be expected if the dust emission were becoming optically thick, but higher frequency data are required to confirm this trend. 
SMA2 and SMA3 are the only strong, compact millimeter sources not to be detected at wavelengths longward of $3.4 \mathrm{~mm}$. Between 1.3 and $3.4 \mathrm{~mm}$ the spectral index of SMA2 is $\alpha=4.0 \pm 0.3$, and that of SMA3 is $\alpha=4.3 \pm 0.3$, consistent with optically thin dust emission. Extrapolating these spectral indices to $7 \mathrm{~mm}$, we would have expected to detect SMA2 and SMA3 at the 1.4 and $0.9 \mathrm{mJy}$ level, respectively (amounting to $7 \sigma$ and $4.5 \sigma$ ) if the emission were unresolved at $7 \mathrm{~mm}$. The sizes derived for SMA2 and SMA3 at $1.3 \mathrm{~mm}$ are $2 . " 1 \times 1$." 3 and 2 ! $^{\prime \prime} \times 1$ ! $^{\prime} 9$. If these are the true sizes of the emitting regions, the peak $7 \mathrm{~mm}$ intensity would be diminished by a factor of $\sim 10$ over that predicted here, plausibly explaining the non-detections. The emission denoted VLA3 is only detected at $7 \mathrm{~mm}$, and thus its nature is very uncertain. The non-detection of VLA3 at $1.3 \mathrm{~cm}$ implies that its spectral index is $>2$, and we estimate a $1.3 \mathrm{~mm}$ upper limit of $\sim 150$ mJy suggesting that its spectral index must also be less than $\sim 3$. These limits suggest that VLA3 is predominately due to dust emission, but contribution from a hypercompact $\mathrm{H}$ II region cannot be excluded.

In summary, of the nine compact sources detected in $\mathrm{I}(\mathrm{N})$, all appear to have a dust component. Four of these sources (SMA1b, SMA1c, SMA1d, and SMA4) also show evidence for free-free emission at our current level of sensitivity. Three sources (SMA1b, SMA1d, and SMA2) and possibly a fourth (SMA4) show modest hot core molecular line emission, also suggesting the presence of a central powering source. One source with neither free-free nor hot core emission does appear to be powering an outflow (SMA6). From this circumstantial evidence it appears that at least six of the compact sources may harbor a central source. However, as described by Zinnecker \& Yorke (2007), in deeply embedded regions it is extraordinarily difficult to determine whether a massive protostar or zero age main sequence star (ZAMS) has formed, as this designation is dependent upon detecting the source in the near-IR (a wavelength regime in which $\mathrm{I}(\mathrm{N})$ is still obscured). In principle, the detection of free-free emission is often taken as indicative of at least an early B-type star. However, even the presence of free-free emission is ambiguous since the accretion luminosity itself can be sufficient to ionize the gas around a protostar. Thus, while it is clear that a number of massive to intermediate stars are forming in $\mathrm{I}(\mathrm{N})$, the exact number and evolutionary state is uncertain.

A few comments on the previously published flux measurements for these sources are in order. When the more accurate absolute flux calibration presented here for the original Carral et al. (2002) $3.6 \mathrm{~cm}$ data is taken into account, the agreement for SMA4 is quite good. However, it is unclear why the detection of SMA1b was not reported by these authors, since it is 1.6 times brighter than SMA4. We obtain $7 \mathrm{~mm}$ flux densities between 2.2 (SMA6) to 8 (SMA1a) times smaller than those reported by Rodríguez et al. (2007), though our $1.3 \mathrm{~cm}$ flux densities are comparable. This discrepancy comes in spite of the fact that the flux densities derived for the phase calibrators at both 7 and $1.3 \mathrm{~cm}$ are within $2 \%$ for our reduction compared to that reported in Rodríguez et al. (2007). As described in \$2.2.3, continuum emission from the strong ultracompact $\mathrm{H}$ II region in source I causes significant contamination of the $I(N)$ region if not included in the cleaning; however our detailed tests suggest that at most it could erroneously increase the integrated fluxes by a factor of $\sim 2$, and thus cannot account for the large discrepancy. In addition, the integrated flux densities reported in Rodríguez et al. (2007) do not appear consistent with the contour levels plotted in their Figures 2 and 3, indeed the peak values are reasonably consistent with those reported here so the problem may have occurred in their integrated Gaussian fits. Finally, our re-imaging of the $3.4 \mathrm{~mm}$ data from Beuther et al. (2008) has increased the peak flux densities by a factor of about 1.5 due to our self-calibration and correction for the primary beam attenuation.

\subsubsection{Mass estimates from the dust emission}

In Table 6 we give estimates for the gas mass $M_{\text {gas }}$, molecular hydrogen column density $N_{H_{2}}$, and molecular hydrogen density $n_{H_{2}}$ for all seven $1.3 \mathrm{~mm}$ dust sources. It should be noted that if a protostar or zero age main sequence star has already formed, the millimeter data is only sensitive to the circum-(proto)stellar material. The gas masses were calculated from

$$
M_{\text {gas }}=\frac{3.24 \times 10^{-3} S_{\nu}(\mathrm{Jy}) D^{2}(\mathrm{kpc}) R C_{\tau_{\text {dust }}}}{J\left(\nu, T_{\text {dust }}\right) \nu^{3}(\mathrm{THz}) \kappa_{\nu}},
$$

where $S_{\nu}$ is the integrated flux density from Table $1, D$ is the distance (assumed to be $1.7 \mathrm{kpc}$ ), $R$ is the gas to dust ratio (assumed to be 100$), J(\nu, T)=1 /\left(\exp \left(h \nu / T_{\text {dust }} k\right)-1\right)$, and $C_{d u s t}$ is the correction factor for the dust opacity $C_{\tau_{\text {dust }}}=\tau_{\text {dust }} /(1-$ $\left.e^{-\tau_{\text {dust }}}\right)$. We have used $\kappa_{1.3 \mathrm{~mm}}=1 \mathrm{~cm}^{2} \mathrm{~g}^{-1}$ which is the average of the dust opacities derived by Ossenkopf \& Henning (1994) for the case of thin ice mantles and densities between $10^{6}$ to $10^{8} \mathrm{~cm}^{-3}$ at $1.3 \mathrm{~mm}$. We have estimated a range of dust temperatures based on the observed spectral line properties of each source: we use the derived $T_{\text {rot }}$ values calculated in $\$ 4.4$ for SMA1 and SMA2, and a lower temperature range for SMA3 to SMA7 based on the non-detection of hot core spectral lines towards these sources. The column densities were estimated from

$$
N_{H_{2}}=\frac{2.4 \times 10^{30} S_{\nu}(\mathrm{Jy}) R C_{\tau_{\text {dust }}}}{\left.J\left(\nu, T_{\text {dust }}\right) \nu^{3}(\mathrm{THz}) \theta_{1}\left({ }^{\prime \prime}\right) \theta_{2}{ }^{\prime \prime}\right) \kappa_{\nu}} \mathrm{cm}^{-2},
$$

where $\theta_{1}$ and $\theta_{2}$ are the fitted sizes from Table 1 . The $n_{H_{2}}$ was calculated by dividing $N_{H_{2}}$ by the geometric mean of the fitted size, assuming a distance of $1.7 \mathrm{kpc}$. The estimated dust masses are similar to those reported in Hunter et al. (2006) when the differences in absolute flux calibration and assumed dust temperatures are taken into account. As with all such estimates, the $M_{\text {gas }}, N_{H_{2}}, n_{H_{2}}$ reported in Table 6 are uncertain to at least a factor of 2 . The total mass estimated for the compact emission of $\mathrm{I}(\mathrm{N})$ is between 89 and $232 \mathrm{M}_{\odot}$. These dust emission based masses do not include the mass of any protostars or ZAMSs that may already have formed within the compact cores. It is also notable that Matthews et al. (2008) estimate that the mass of the whole $\mathrm{I}(\mathrm{N})$ core is $\sim 2780 \mathrm{M}_{\odot}$, so there is still a considerable reservoir of material that has not yet collapsed.

The values for SMA1 are particularly uncertain because the current $1.3 \mathrm{~mm}$ resolution is insufficient to distinguish between the four dust sources detected at $7 \mathrm{~mm}$ (SMA1a, SMA1b, SMA1c, and SMA1d). This unresolved structure makes the apparent size of this source quite large - significantly affecting all derived quantities. For example, if we assume that the majority of dust emission comes from a region half the size (in both directions) of the current fit $\left(2 !^{\prime \prime} 8 \times 1{ }^{\prime \prime} 9\right)$, the mass increases only a little (due to increase in $C_{\tau_{\text {dust }}}$ ), but $N_{\mathrm{H}_{2}}$ and $n_{\mathrm{H}_{2}}$ both increase by about an order of magnitude. An additional source of uncertainty is the $T_{\text {dust }}$. As described in $\S 4.3$, the hot core line emission appears to arise 
from a $0 .{ }^{\prime \prime} 8$ region encompassing only SMA1b and SMA1d. Thus, the $T_{\text {dust }}$ range derived from the $\mathrm{CH}_{3} \mathrm{OH}$ rotation diagram may not be appropriate for SMA1a or SMA1c. Using $\kappa_{7} \mathrm{~mm}=0.05 \mathrm{~cm}^{2} \mathrm{~g}^{-1}$ (obtained by scaling $\kappa_{1.3 \mathrm{~mm}}=1$ by $(7 \mathrm{~mm} / 1.3 \mathrm{~mm})^{-\beta}$ with $\beta=\alpha_{\text {dust }}-2$ where $\alpha_{\text {dust }}=3.8$ from Fig. 13), and $T_{d u s t}=155-175 \mathrm{~K}$ we find that the sum of the masses derived for SMA1a,b,c, and d at $7 \mathrm{~mm}$ is $20-18 \mathrm{M}_{\odot}$ in good agreement with the $1.3 \mathrm{~mm}$ estimate for SMA1 (16 - $14 \mathrm{M}_{\odot}$ ). Indeed, the agreement is nearly perfect if we assume that the dust emission at $1.3 \mathrm{~mm}$ arises from an area $5 \times$ smaller than the current size estimate (i.e. Table 1), which is certainly plausible. Higher resolution $1.3 \mathrm{~mm}$ observations are required to better constrain the temperatures of SMA1a and SMA1c, as well as the sizes of all four SMA1 sources.

\subsection{Evidence for Accretion and Infall Around SMA1?}

As described in $\$ 3.4$, a SE-NW velocity gradient of $\sim$ $5 \mathrm{~km} \mathrm{~s}^{-1}$ is observed toward SMA1 in some (but not all) of the species with compact emission (a similar gradient was inferred from a double peaked $\mathrm{NH}_{3}(6,6)$ profile by Beuther et al. 2007). Since the gradient is barely spatially resolved with the current SMA resolution, the nature of this gradient is unclear. Its direction is more or less perpendicular to the large scale NE-SW outflow suggestive of a possible disk interpretation, but it also has the same orientation as a line joining the SMA1b and SMA1d sources, and thus could simply represent unresolved emission from these two sources if they have different systemic velocities (as was found for CephA-East in a similar situation by Brogan et al. 2007). We note that this velocity gradient was not observed in the $\mathrm{CH}_{3} \mathrm{CN}\left(5_{K}-4_{K}\right)$ transitions observed by Beuther et al. (2008) with the ATCA. Higher spatial resolution data will be needed to further explore this velocity gradient.

As described in $\$ 3.5 .2$, there is a trend of increasingly redshifted absorption with increasing line excitation temperature toward SMA1, using $\mathrm{CN}\left(E_{l}=3.8 \mathrm{~cm}^{-1}\right), \mathrm{H}_{2} \mathrm{CO}\left(E_{l}=7 \mathrm{~cm}^{-1}\right)$, and $\mathrm{H}_{2} \mathrm{CO}\left(E_{l}=40 \mathrm{~cm}^{-1}\right)$ as tracers (see Fig. $\left.10 \mathrm{a}\right)$. In $\$ 4.3$ we find that the hot core of SMA1 has a temperature of $165 \pm 9 \mathrm{~K}$, demonstrating that this source is definitely centrally heated, suggesting higher excitation lines will be found closer to the center. We suggest that the observed redshift/temperature trend is tracing infall that is accelerating with depth into the core. It would be very interesting to follow-up this tentative result with higher angular and spectral resolution. In particular if the continuum emission were resolved, one could be assured that all emission/absorption originates from only the front side of the source, removing any ambiguities (see for example Chandler et al. 2005).

\subsection{The Location of the Protocluster}

Finally, it is interesting to note that the massive protocluster appears to be forming near the northwestern edge of the parent cloud rather than toward the center. This offset is apparent in both the JCMT $450 \mu \mathrm{m}$ continuum image (Fig. 4.3) and in the ammonia $(1,1)$ and $(2,2)$ images (Beuther et al. 2005). It is notable that the archival SCUBA $450 \mu \mathrm{m}$ images recently produced by Di Francesco et al. (2008) show very good positional agreement (to within $3^{\prime \prime}$ ) with the earlier images produced by Sandell (2000) (and shown in Fig.4.3). The ridge of dust emission between the protocluster and the masers associated with the SM2 continuum source coincides with a dark area in the Spitzer 3-color image (Fig. 11), i.e. an infrared dark cloud (IRDC; see for example Egan et al. 1998). Clearly this region is optically-thick in the mid-infrared, and contains a significant amount of potential star-forming material. Future observations with broader bandwidth and greater sensitivity with the EVLA and ALMA may reveal whether this area is truly quiescent or contains embedded, pre-protostellar cores.

\section{CONCLUSIONS}

Our multiwavelength radio through near-infrared investigation of the massive protocluster NGC6334I(N) provides new morphological and quantitative detail on the star formation activity of the individual massive cores. We show that $\mathrm{I}(\mathrm{N})$ is undergoing copious massive star formation with many of the dominant sources detected only at millimeter wavelengths. The principal source SMA1 is resolved into four components of dust and/or free-free emission (SMA1a, b, c, and d). Delineated by centimeter continuum and water masers, SMA1b and SMA1d form a close central pair ( $\gtrsim 800$ AU) which powers a hot core $\left(T_{r o t}=165 \pm 9 \mathrm{~K}\right)$ of molecular line emission and likely drives the dominant large-scale bipolar outflow which exhibits evidence of precession. We find a spatially unresolved $\sim 5 \mathrm{~km} \mathrm{~s}^{-1}$ velocity gradient across SMA1 (encompassing all 4 subarcsecond components). The origin of this velocity gradient (rotation, or cluster kinematics) requires higher resolution millimeter wavelength follow-up. We also detect possible infall that is accelerating with depth into the SMA1 core; higher angular resolution is also required to confirm this finding. The mass of gas around SMA1 as traced by compact emission is estimated to be $\sim 15 \mathrm{M}_{\odot}$, but if this core is still actively accreting, this may grow significantly larger in the future.

A weaker hot core $\left(T_{r o t}=145 \pm 12 \mathrm{~K}\right)$ is found toward SMA2 which also exhibits redshifted $\mathrm{CN}$ absorption along with with highly blueshifted water masers, suggesting simultaneous infall and outflow in this source. SMA4 consists of a dust core with a probable centimeter jet, water maser emission, and blueshifted $\mathrm{SiO}$ emanating to the west suggesting an outflow which terminates in a reflection nebula traced by the $24 \mu \mathrm{m}$ source. Mostly lacking in molecular lines, SMA6 exhibits a centimeter-millimeter SED consistent with pure dust emission and drives an outflow traced by $\mathrm{SiO}, \mathrm{CO}$ and $44 \mathrm{GHz}$ methanol masers. Overall, the narrow velocity range and spatial arrangement of the $44 \mathrm{GHz}$ methanol masers with respect to the protocluster members are consistent with their origin in low-velocity gas impacted by outflows from these objects.

The presence of multiple outflows in the region suggests that many protostars are forming simultaneously. At the same time, the variety of phenomena associated with the individual cluster members suggests a spread in either age or total luminosity. Also of interest is the spread in systemic velocities observed between the cluster members, this spread is at least $2.5 \mathrm{~km} \mathrm{~s}^{-1}$ and may provide an important clue about the dynamics of cluster formation. A more quantitative assessment of these key properties will require broader wavelength coverage to better constrain the SEDs, and more sensitive continuum and line observations enabled by the EVLA and ALMA in the near future. At the same time, a deeper exploration of the mostly featureless, infrared-dark region between the protocluster and SM2 may prove fruitful in the study of preprotostellar cores.

We thank Göran Sandell for providing us with the calibrated JCMT $450 \mu \mathrm{m}$ image. This research has made use of the NASA's Astrophysics Data System Bibliographic Services, 
the Cologne Database for Molecular Spectroscopy, Splata- logue, and the JPL line catalog.

\section{REFERENCES}

Anglada, G., López, R., Estalella, R., Masegosa, J., Riera, A., \& Raga, A. C. 2007, AJ, 133, 2799

Araya, E., Hofner, P., Kurtz, S., Olmi, L., \& Linz, H. 2008, ApJ, 675, 420

Avalos, M., Lizano, S., Rodríguez, L. F., Franco-Hernández, R., \& Moran, J. M. 2006, ApJ, 641, 406

Beuther, H., Thorwirth, S., Zhang, Q., Hunter, T. R., Megeath, S. T., Walsh, A. J., \& Menten, K. M. 2005, ApJ, 627, 834

Beuther, H., Walsh, A. J., Thorwirth, S., Zhang, Q., Hunter, T. R., Megeath, S. T., \& Menten, K. M. 2007, A\&A, 466, 989

- 2008, ArXiv e-prints, 801

Blundell, R. et al. 1998, in Institute of Electrical and Electronics Engineers, Inc. Conference, 246-247

Brogan, C. L., Chandler, C. J., Hunter, T. R., Shirley, Y. L., \& Sarma, A. P. 2007, ApJ, 660, L133

Carey, S. J., Noriega-Crespo, A., Mizuno, D. R., Shenoy, S., Paladini, R., Kraemer, K. E., Price, S. D., Flagey, N., Ryan, E., Ingalls, J. G., Kuchar, T. A., Pinheiro Gonçalves, D., Indebetouw, R., Billot, N., Marleau, F. R., Padgett, D. L., Rebull, L. M., Bressert, E., Ali, B., Molinari, S., Martin, P. G., Berriman, G. B., Boulanger, F., Latter, W. B., Miville-Deschenes, M. A., Shipman, R., \& Testi, L. 2009, PASP, 121, 76

Carral, P., Kurtz, S. E., Rodríguez, L. F., Menten, K., Cantó, J., \& Arceo, R. 2002, AJ, 123, 2574

Caswell, J. L. 2009, arXiv:0907.5255

Chandler, C. J., Brogan, C. L., Shirley, Y. L., \& Loinard, L. 2005, ApJ, 632, 371

Cheung, L., Frogel, J. A., Hauser, M. G., \& Gezari, D. Y. 1978, ApJ, 226 L149

Cragg, D. M., Sobolev, A. M., \& Godfrey, P. D. 2002, MNRAS, 331, 521

-. 2005, MNRAS, 360, 533

Cunningham, N. J., Moeckel, N., \& Bally, J. 2009, ApJ, 692, 943

Cyganowski, C. J., Brogan, C. L., \& Hunter, T. R. 2007, AJ, 134, 346

Cyganowski, C. J., Brogan, C. L., Hunter, T. R., \& Churchwell, E. 2007, ApJ, 702, 1615

Cyganowski, C. J., Whitney, B. A., Holden, E., Braden, E., Brogan, C. L., Churchwell, E., Indebetouw, R., Watson, D. F., Babler, B. L., Benjamin, R., Gomez, M., Meade, M. R., Povich, M. S., Robitaille, T. P., \& Watson, C. $2008, \mathrm{AJ}, 136,2391$

De Buizer, J. 2007, in IAU Symposium, Vol. 242, IAU Symposium, ed. J. M. Chapman \& W. A. Baan, 102-109

De Buizer, J. M., Osorio, M., \& Calvet, N. 2005, ApJ, 635, 452

Di Francesco, J., Johnstone, D., Kirk, H., MacKenzie, T., \& Ledwosinska, E. 2008, ApJS, 175, 277

Egan, M. P., Shipman, R. F., Price, S. D., Carey, S. J., Clark, F. O., \& Cohen, M. 1998, ApJ, 494, L199+

Ellison, B. N., Schaffer, P. L., Schaal, W., Miller, R. E., \& Vail, D. 1989, International Journal of Infrared and Millimeter Waves, 10, 937

Fazio, G. G. et al. 2004, ApJS, 154, 10

Feigelson, E. D., Martin, A. L., McNeill, C. J., Broos, P. S., \& Garmire, G. P. 2009, ArXiv e-prints

Gezari, D. Y. 1982, ApJ, 259, L29

Goldsmith, P. F. \& Langer, W. D. 1999, ApJ, 517, 209

Haschick, A. D., Menten, K. M., \& Baan, W. A. 1990, ApJ, 354, 556

Hofner, P. \& Churchwell, E. 1996, A\&AS, 120, 283

Hunter, T. R., Brogan, C. L., Indebetouw, R., \& Cyganowski, C. J. 2008, ApJ, 680, 1271

Hunter, T. R., Brogan, C. L., Megeath, S. T., Menten, K. M., Beuther, H., \& Thorwirth, S. 2006, ApJ, 649, 888

Hunter, T. R., Testi, L., Zhang, Q., \& Sridharan, T. K. 1999, AJ, 118, 477

Kogan, L. \& Slysh, V. 1998, ApJ, 497, 800

Kuiper, T. B. H., Peters, W. L., Forster, J. R., Gardner, F. F., \& Whiteoak, J. B. 1995, ApJ, 446, 692

Kurtz, S., Hofner, P., \& Álvarez, C. V. 2004, ApJS, 155, 149

Le Bourlot, J., Pineau des Forêts, G., \& Flower, D. R. 1999, MNRAS, 305, 802

Le Bourlot, J., Pineau des Forêts, G., Flower, D. R., \& Cabrit, S. 2002, MNRAS, 332, 985

Leurini, S., Schilke, P., Wyrowski, F., \& Menten, K. M. 2007, A\&A, 466, 215
Lizano, S. 2008, in Astronomical Society of the Pacific Conference Series, Vol. 387, Massive Star Formation: Observations Confront Theory, ed. H. Beuther, H. Linz, \& T. Henning, 232-+

Matthews, H. E., McCutcheon, W. H., Kirk, H., White, G. J., \& Cohen, M 2008, AJ, 136, 2083

Megeath, S. T. \& Tieftrunk, A. R. 1999, ApJ, 526, L113

Minier, V., Ellingsen, S. P., Norris, R. P., \& Booth, R. S. 2003, A\&A, 403, 1095

Moran, J. M. \& Rodriguez, L. F. 1980, ApJ, 236, L159

Müller, H. S. P., Thorwirth, S., Roth, D. A., \& Winnewisser, G. 2001, A\&A, 370, L49

Neckel, T. 1978, A\&A, 69, 5

Neufeld, D. A. \& Yuan, Y. 2008, ApJ, 678, 974

Ossenkopf, V. \& Henning, T. 1994, A\&A, 291, 943

Persi, P. \& Tapia, M. 2008, Star Formation in NGC 6334, ed. B. Reipurth, 456-+

Pickett, H. M., Poynter, I. R. L., Cohen, E. A., Delitsky, M. L., Pearson,

J. C., \& Muller, H. S. P. 1998, Journal of Quantitative Spectroscopy and Radiative Transfer, 60, 883

Pirogov, L., Zinchenko, I., Caselli, P., Johansson, L. E. B., \& Myers, P. C. 2003, A\&A, 405, 639

Plambeck, R. L. \& Menten, K. M. 1990, ApJ, 364, 555

Pratap, P., Shute, P. A., Keane, T. C., Battersby, C., \& Sterling, S. 2008, AJ, 135,1718

Reach, W. T., Megeath, S. T., Cohen, M., Hora, J., Carey, S., Surace, J., Willner, S. P., Barmby, P., Wilson, G., Glaccum, W., Lowrance, P., Marengo, M., \& Fazio, G. G. 2005, PASP, 117, 978

Reynolds, S. P. 1986, ApJ, 304, 713

Robitaille, T. P., Whitney, B. A., Indebetouw, R., \& Wood, K. 2007, ApJS, 169,328

Rodríguez, L. F., Zapata, L. A., \& Ho, P. T. P. 2007, ApJ, 654, L143

Sandell, G. 2000, A\&A, 358, 242

Sandell, G., Goss, W. M., \& Wright, M. 2005, ApJ, 621, 839

Scoville, N. Z. \& Kwan, J. 1976, ApJ, 206, 718

Skrutskie, M. F., Cutri, R. M., Stiening, R., Weinberg, M. D., Schneider, S., Carpenter, J. M., Beichman, C., Capps, R., Chester, T., Elias, J., Huchra, J., Liebert, J., Lonsdale, C., Monet, D. G., Price, S., Seitzer, P., Jarrett, T., Kirkpatrick, J. D., Gizis, J. E., Howard, E., Evans, T., Fowler, J., Fullmer, L., Hurt, R., Light, R., Kopan, E. L., Marsh, K. A., McCallon, H. L., Tam, R., Van Dyk, S., \& Wheelock, S. 2006, AJ, 131, 1163

Slysh, V. I., Kalenskii, S. V., Valtts, I. E., \& Otrupcek, R. 1994, MNRAS, 268,464

Smith, H. A., Hora, J. L., Marengo, M., \& Pipher, J. L. 2006, ApJ, 645, 1264 Smith, M. D. 1995, A\&A, 296, 789

Smith, M. D. \& Rosen, A. 2005, MNRAS, 357, 1370

Straw, S. M. \& Hyland, A. R. 1989, ApJ, 340, 318

Sutton, E. C., Sobolev, A. M., Salii, S. V., Malyshev, A. V., Ostrovskii, A. B., \& Zinchenko, I. I. 2004, ApJ, 609, 231

Thorwirth, S., Winnewisser, G., Megeath, S. T., \& Tieftrunk, A. R. 2003, in ASP Conf. Ser. 287: Galactic Star Formation Across the Stellar Mass Spectrum, 257-260

Tofani, G., Felli, M., Taylor, G. B., \& Hunter, T. R. 1995, A\&AS, 112, 299

Val'Tts, I. E. \& Larionov, G. M. 2007, Astronomy Reports, 51, 519

van der Tak, F. F. S., Black, J. H., Schöier, F. L., Jansen, D. J., \& van

Dishoeck, E. F. 2007, A\&A, 468, 627

van Dishoeck, E. F. \& Blake, G. A. 1998, ARA\&A, 36, 317

Walsh, A. J., Burton, M. G., Hyland, A. R., \& Robinson, G. 1998, MNRAS, 301,640

Wolniewicz, L., Simbotin, I., \& Dalgarno, A. 1998, ApJS, 115, 293

Wrathmall, S. A., Gusdorf, A., \& Flower, D. R. 2007, MNRAS, 382, 133

Ybarra, J. E. \& Lada, E. A. 2009, ApJ, 695, L120

Zhang, Q., Hunter, T. R., Sridharan, T. K., \& Cesaroni, R. 1999, ApJ, 527, L117

Zinnecker, H., \& Yorke, H. W. 2007, ARA\&A, 45, 481 
TABLE 1

OBSERVED PROPERTIES OF COMPACT CONTINUUM SOURCES

\begin{tabular}{|c|c|c|c|c|c|}
\hline \multirow[b]{2}{*}{ Source } & \multicolumn{2}{|c|}{ Centroid position $^{a}$} & \multirow{2}{*}{$\begin{array}{c}\text { Peak }^{b} \\
\text { Intensity } \\
\left(\mathrm{mJy} \mathrm{beam}^{-1}\right)\end{array}$} & \multirow{2}{*}{$\begin{array}{l}\text { Integrated }^{b} \\
\text { Flux Density } \\
\quad(\mathrm{mJy})\end{array}$} & \multirow{2}{*}{$\begin{array}{c}\text { Estimated } \\
\text { Size } \\
\left({ }^{\prime \prime} \times{ }^{\prime \prime}\left[\text { P.A. }{ }^{\circ}\right]\right)\end{array}$} \\
\hline & $\begin{array}{l}\mathrm{RA}(\mathrm{J} 2000) \\
\alpha\left(\begin{array}{lll}\mathrm{h} & \mathrm{m}\end{array}\right)\end{array}$ & $\begin{array}{c}\operatorname{Dec}(\mathrm{J} 2000) \\
\delta\left({ }^{\circ},{ }^{\prime} \prime\right)\end{array}$ & & & \\
\hline \multicolumn{6}{|c|}{ 1.3 Millimeter $^{c}$} \\
\hline SMA1 & 172055.188 & -354503.92 & $826(6)$ & $2550(50)$ & $2.8 \times 1.9[23]$ \\
\hline SMA2 & 172054.890 & -354506.71 & $324(6)$ & $980(25)$ & $2.1 \times 1.3[137]$ \\
\hline SMA3 & 172054.990 & -354507.29 & $258(6)$ & $1060(31)$ & $2.5 \times 1.9[9]$ \\
\hline SMA4 & 172054.667 & -354508.38 & $141(6)$ & $770(40)$ & $3.0 \times 1.5[41]$ \\
\hline SMA5 & 172055.054 & -354501.49 & $140(6)$ & $440(100)$ & $\leq 2.0$ \\
\hline SMA6 & 172054.563 & -354517.71 & $282(9)$ & $600(29)$ & $2.2 \times 0.9[38]$ \\
\hline SMA7 & 172054.931 & -354457.79 & $60(6)$ & $360(40)$ & $3.2 \times 2.3[47]$ \\
\hline \multicolumn{6}{|c|}{ 3.4 Millimeter $^{e, f}$} \\
\hline SMA1 & 172055.194 & -354503.79 & $55(5)$ & $90(10)$ & $\leq 3.0$ \\
\hline SMA2 & 172054.86 & -354506.5 & $24(5)$ & $\ldots$ & $\overline{<} 1.6$ \\
\hline SMA3 & 172054.99 & -354507.0 & $20(5)$ & $\cdots$ & $<1.6$ \\
\hline SMA $4^{d}$ & 172054.69 & -354507.5 & $13(3)$ & $\cdots$ & $<1.6$ \\
\hline $\mathrm{SMA}^{d}$ & 172055.04 & -354501.3 & $15(3)$ & $\ldots$ & $<1.6$ \\
\hline SMA6 & 172054.588 & -354517.86 & $31(3)$ & $\cdots$ & $<1.6$ \\
\hline \multicolumn{6}{|c|}{7 Millimeter } \\
\hline SMA1a & 172055.150 & -354505.28 & $0.9(0.2)$ & $1.7(0.3)$ & $\leq 1$ \\
\hline SMA1b & 172055.194 & -354503.50 & $1.3(0.2)$ & $2.5(0.4)$ & $0.6 \times \overline{0} .4[130]$ \\
\hline SMA1c & 172055.275 & -354502.46 & $0.9(0.2)$ & $\cdots$ & $<0.4$ \\
\hline 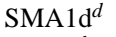 & 172055.229 & -354503.68 & $1.0(0.2)$ & $\cdots$ & $<0.4$ \\
\hline $\mathrm{VLA}^{d}$ & 172054.999 & -354506.48 & $1.0(0.2)$ & $1.8(0.3)$ & $\leq 1$ \\
\hline SMA $4^{d}$ & 172054.624 & -354508.24 & $0.7(0.2)$ & $\cdots$ & $<0.4$ \\
\hline SMA6 & 172054.585 & -354516.97 & $1.3(0.2)$ & $1.7(0.3)$ & $0.9 \times 0.7[15]$ \\
\hline \multicolumn{6}{|c|}{1.3 Centimeter } \\
\hline SMA1a & $\cdots$ & $\cdots$ & $<0.18(0.06)$ & $\cdots$ & $\cdots$ \\
\hline SMA1b & 172055.191 & -354503.53 & $0.50(0.07)$ & $\cdots$ & $<0.3$ \\
\hline SMA1c & 172055.270 & -354502.45 & $0.27(0.06)$ & $\cdots$ & $<0.4$ \\
\hline $\mathrm{SMA}_{1 \mathrm{~d}}{ }^{d}$ & 172055.229 & -354503.68 & $0.30(0.06)$ & $\cdots$ & $<0.3$ \\
\hline SMA4 & 172054.623 & -354508.25 & $0.47(0.07)$ & $\cdots$ & $<0.3$ \\
\hline SMA6 & $\ldots$ & $\ldots$ & $<0.18(0.06)$ & $\cdots$ & $\ldots$ \\
\hline \multicolumn{6}{|c|}{3.6 Centimeter $^{e}$} \\
\hline SMA1a & $\cdots$ & $\cdots$ & $<0.12(0.04)$ & $\cdots$ & $\cdots$ \\
\hline $\mathrm{SMA} 1 \mathrm{~b}^{d}$ & 172055.202 & -354503.74 & $0.39(0.04)$ & $\cdots$ & $<0.3$ \\
\hline SMA1c & $\cdots$ & $\cdots$ & $<0.12(0.04)$ & $\cdots$ & $\ldots$ \\
\hline SMA1d & $\cdots$ & $\cdots$ & $<0.12(0.04)$ & $\cdots$ & $\cdots$ \\
\hline SMA4 & 172054.623 & -354508.25 & $0.25(0.04)$ & $\cdots$ & $<0.3$ \\
\hline SMA6 & $\cdots$ & $\cdots$ & $<0.12(0.04)$ & $\cdots$ & $\cdots$ \\
\hline
\end{tabular}

a Relative uncertainties are indicated by the number of significant figures shown. Absolute uncertainties are at least an order of magnitude larger.

b Because the data have different resolutions, the peak intensity values should only be compared for cases where the source is unresolved (indicated by $\cdots$ symbols in Integrated Flux Density column).Estimated statistical uncertainties indicated in parenthesis.

${ }^{c}$ Fits based on uniformly weighted image. SMA1, SMA4, and SMA6 appear to also have a more compact core so the fitted peaks are underestimated.

d New detection based on archival data.

e Primary beam correction makes a significant difference for these images; the original papers do not appear to have performed this correction based on reported flux densities.

${ }^{\mathrm{f}}$ Extra uncertainty for the peak and integrated fluxes for SMA1 and SMA5, as well as SMA2 and SMA3 were added because these source pairs were barely resolved from each other. 
TABLE 2

22.235 GHz Water MASER Groups IN NGC 6334 I(N)

\begin{tabular}{|c|c|c|c|c|c|c|}
\hline \multirow[b]{2}{*}{ \# } & \multicolumn{2}{|c|}{ Centroid position $^{a}$} & \multirow{2}{*}{$\begin{array}{l}\text { Associated } \\
\text { mm source } \\
\text { (offset) }\end{array}$} & \multirow{2}{*}{$\begin{array}{c}\text { Peak } \\
\text { Intensity } \\
\left(\mathrm{Jy} \mathrm{beam}^{-1}\right)\end{array}$} & \multirow{2}{*}{$\begin{array}{c}\text { Peak } \\
\text { Velocity } \\
\left(\mathrm{km} \mathrm{s}^{-1}\right)\end{array}$} & \multirow{2}{*}{$\begin{array}{c}\text { Velocity } \\
\text { Range } \\
\left(\mathrm{km} \mathrm{s}^{-1}\right)\end{array}$} \\
\hline & $\begin{array}{c}\mathrm{RA}(\mathrm{J} 2000) \\
\alpha\left(\begin{array}{lll}\mathrm{h} & \mathrm{m} & \mathrm{s}\end{array}\right)\end{array}$ & $\begin{array}{c}\operatorname{Dec}(\mathrm{J} 2000) \\
\delta\left({ }^{\circ}, \prime \prime\right)\end{array}$ & & & & \\
\hline 1 & 172056.058 & -354532.59 & $\mathrm{SM} 2^{\mathrm{b}}\left(4.50^{\prime \prime}\right)$ & 38.3 & -6.0 & +5.2 to -16.2 \\
\hline 2 & 172055.650 & -354532.48 & $\mathrm{SM} 2^{\mathrm{b}}\left(1.78^{\prime \prime}\right)$ & 9.2 & -9.0 & +7.2 to -15.6 \\
\hline 3 & 172054.600 & -354517.27 & SMA6 $\left(0.67^{\prime \prime}\right)$ & 8.8 & -2.2 & +10.4 to -12.6 \\
\hline 4 & 172054.152 & -354513.66 & - & 29.0 & -2.7 & -0.7 to -4.4 \\
\hline 5 & 172054.618 & -354508.65 & SMA4 $\left(2.11^{\prime \prime}\right)$ & 17.7 & +0.2 & +12.4 to $-12.9^{\mathrm{c}}$ \\
\hline 6 & 172054.870 & -354506.32 & SMA2 $\left(0.55^{\prime \prime}\right)$ & 2.7 & -13.3 & -4.7 to -15.6 \\
\hline 7 & 172054.819 & -354506.18 & SMA2 $\left(1.11^{\prime \prime}\right)$ & 5.3 & -0.7 & +0.2 to -1.7 \\
\hline 8 & 172055.192 & -354503.77 & SMA1b $\left(0.24^{\prime \prime}\right)$ & 78.1 & -6.3 & +13.1 to $-26.8^{\mathrm{d}}$ \\
\hline 9 & 172055.228 & -354503.66 & SMA1d $\left(0.02^{\prime \prime}\right)$ & 75.7 & +3.9 & +5.2 to +2.2 \\
\hline 10 & 172055.147 & -354503.78 & SMA1 $\left(0.53^{\prime \prime}\right)$ & 3.4 & -8.6 & -7.7 to -9.6 \\
\hline 11 & 172055.215 & -354502.02 & SMA1 $\left(1.96^{\prime \prime}\right)$ & 5.5 & -4.4 & -3.7 to -6.3 \\
\hline
\end{tabular}

a Intensity weighted positions for masers listed under each group name in Table 3

b See Sandell (2000).

${ }^{\mathrm{c}}$ Emission extends beyond the positive velocity extent of the bandpass.

${ }^{\mathrm{d}}$ Emission extends beyond the negative velocity extent of the bandpass. 
TABLE 3

22.235 GHz Water Masers Detected in NGC $6334 \mathrm{I}(\mathrm{N})$

\begin{tabular}{|c|c|c|c|}
\hline \multirow{2}{*}{$\begin{array}{l}\text { Velocity } \\
\left(\mathrm{km} \mathrm{s}^{-1}\right)\end{array}$} & \multicolumn{2}{|c|}{ Centroid position } & \multirow{2}{*}{$\begin{array}{c}\text { Peak } \\
\text { Intensity } \\
\left(\mathrm{Jy} \mathrm{beam}^{-1}\right)\end{array}$} \\
\hline & $\begin{array}{l}\text { R.A. (J2000) } \\
\alpha\left(\begin{array}{ll}(\mathrm{h} \mathrm{m} \mathrm{s})\end{array}\right.\end{array}$ & $\begin{array}{c}\operatorname{Dec}(\mathrm{J} 2000) \\
\delta\left({ }^{\circ},{ }^{\prime} \prime\right)\end{array}$ & \\
\hline \multicolumn{4}{|c|}{ Group C1 } \\
\hline+5.2 & 172056.0583 & -354532.4934 & 0.384 \\
\hline+4.9 & 172056.0599 & -354532.5678 & 0.548 \\
\hline+4.5 & 172056.0585 & -354532.5920 & 0.586 \\
\hline+4.2 & 172056.0578 & -354532.5805 & 0.893 \\
\hline+3.9 & 172056.0581 & -354532.5797 & 1.373 \\
\hline+3.5 & 172056.0586 & -354532.5776 & 1.764 \\
\hline+3.2 & 172056.0579 & -354532.6134 & 1.675 \\
\hline+2.9 & 172056.0580 & -354532.5594 & 1.534 \\
\hline+2.5 & 172056.0581 & -354532.5946 & 1.185 \\
\hline+2.2 & 172056.0584 & -354532.5566 & 0.934 \\
\hline+1.9 & 172056.0584 & -354532.5619 & 0.639 \\
\hline+1.6 & 172056.0614 & -354532.4557 & 0.508 \\
\hline+1.2 & 172056.0617 & -354532.4066 & 0.381 \\
\hline+0.9 & 172056.0555 & -354532.5404 & 0.535 \\
\hline+0.6 & 172056.0579 & -354532.5826 & 0.504 \\
\hline+0.2 & 172056.0590 & -354532.5242 & 0.497 \\
\hline-0.1 & 172056.0607 & -354532.4663 & 0.422 \\
\hline-0.4 & 172056.0584 & -354532.5610 & 0.400 \\
\hline-0.7 & 172056.0597 & -354532.5347 & 0.558 \\
\hline-1.1 & 172056.0566 & -354532.6202 & 0.652 \\
\hline-1.4 & 172056.0588 & -354532.5413 & 0.578 \\
\hline-1.7 & 172056.0581 & -354532.6271 & 0.749 \\
\hline-2.1 & 172056.0587 & -354532.5695 & 1.507 \\
\hline-2.4 & 172056.0582 & -354532.5774 & 3.357 \\
\hline-2.7 & 172056.0580 & -354532.5891 & 6.453 \\
\hline-3.0 & 172056.0581 & -354532.5907 & 11.608 \\
\hline-3.4 & 172056.0579 & -354532.5977 & 16.950 \\
\hline-3.7 & 172056.0579 & -354532.5953 & 13.072 \\
\hline-4.0 & 172056.0579 & -354532.5929 & 10.177 \\
\hline-4.4 & 172056.0579 & -354532.5925 & 13.265 \\
\hline-4.7 & 172056.0577 & -354532.5900 & 16.513 \\
\hline-5.0 & 172056.0578 & -354532.5893 & 20.479 \\
\hline-5.4 & 172056.0578 & -354532.5910 & 28.570 \\
\hline-5.7 & 172056.0579 & -354532.5903 & 38.006 \\
\hline-6.0 & 172056.0578 & -354532.5935 & 38.297 \\
\hline-6.3 & 172056.0578 & -354532.5900 & 28.243 \\
\hline-6.7 & 172056.0577 & -354532.5910 & 19.718 \\
\hline-7.0 & 172056.0578 & -354532.5912 & 22.008 \\
\hline-7.3 & 172056.0578 & -354532.5952 & 31.096 \\
\hline-7.7 & 172056.0577 & -354532.5945 & 35.008 \\
\hline-8.0 & 172056.0579 & -354532.5918 & 28.781 \\
\hline-8.3 & 172056.0579 & -354532.5943 & 18.181 \\
\hline-8.6 & 172056.0580 & -354532.5908 & 10.865 \\
\hline-9.0 & 172056.0582 & -354532.5926 & 7.395 \\
\hline-9.3 & 172056.0582 & -354532.5850 & 4.048 \\
\hline-9.6 & 172056.0582 & -354532.5758 & 1.460 \\
\hline-10.0 & 172056.0600 & -354532.6461 & 0.521 \\
\hline-15.6 & 172056.0558 & -354532.4942 & 0.536 \\
\hline-15.9 & 172056.0566 & -354532.5314 & 0.608 \\
\hline-16.2 & 172056.0558 & -354532.5633 & 0.515 \\
\hline-21.5 & 172056.0600 & -354532.3669 & 0.375 \\
\hline+5.2 & 172056.0585 & -354532.4918 & 0.432 \\
\hline+4.9 & 172056.0597 & -354532.5667 & 0.562 \\
\hline+4.5 & 172056.0587 & -354532.5882 & 0.681 \\
\hline+4.2 & 172056.0579 & -354532.5851 & 1.028 \\
\hline+3.9 & 172056.0582 & -354532.5809 & 1.426 \\
\hline+3.5 & 172056.0586 & -354532.5788 & 1.805 \\
\hline+3.2 & 172056.0579 & -354532.6131 & 1.706 \\
\hline+2.9 & 172056.0580 & -354532.5580 & 1.577 \\
\hline+2.5 & 172056.0581 & -354532.5956 & 1.228 \\
\hline+2.2 & 172056.0584 & -354532.5573 & 0.963 \\
\hline+1.9 & 172056.0583 & -354532.5609 & 0.652 \\
\hline+1.6 & 172056.0613 & -354532.4586 & 0.509 \\
\hline+0.9 & 172056.0556 & -354532.5405 & 0.566 \\
\hline+0.6 & 172056.0580 & -354532.5863 & 0.547 \\
\hline+0.2 & 172056.0595 & -354532.5222 & 0.460 \\
\hline-0.1 & 172056.0603 & -354532.4752 & 0.459 \\
\hline-0.4 & 172056.0583 & -354532.5707 & 0.450 \\
\hline-0.7 & 172056.0600 & -354532.5378 & 0.579 \\
\hline-1.1 & 172056.0565 & -354532.6301 & 0.636 \\
\hline-1.4 & 172056.0588 & -354532.5398 & 0.573 \\
\hline & 172056.0581 & -354532.6371 & 0.745 \\
\hline-2.1 & 172056.0587 & -354532.5701 & 1.514 \\
\hline-2.4 & 172056.0582 & -354532.5774 & 3.370 \\
\hline-2.7 & 172056.0580 & -354532.5890 & 6.416 \\
\hline-3.0 & 172056.0581 & -354532.5906 & 11.555 \\
\hline-3.4 & 172056.0579 & -354532.5976 & 16.873 \\
\hline
\end{tabular}


TABLE 4

44 GHz Class I Methanol masers detected in NGC 6334 I(N)

\begin{tabular}{|c|c|c|c|c|c|c|c|}
\hline \multirow{2}{*}{$\begin{array}{c}\text { Spot } \\
\text { Number }\end{array}$} & \multicolumn{2}{|c|}{ Peak position } & \multirow{2}{*}{$\begin{array}{l}\text { Peak intensity } \\
\left(\mathrm{Jy} \mathrm{beam}^{-1}\right)\end{array}$} & \multirow{2}{*}{$\begin{array}{l}T_{\text {Brightness }} \\
(\mathrm{K})\end{array}$} & Vel & ity (km & $-1)$ \\
\hline & R.A. & Dec & & & $v_{\text {peak }}$ & $v_{\min }$ & $v_{\max }$ \\
\hline 1 & 172053.351 & -354519.31 & $13.48 \pm 0.22$ & 16340 & -3.17 & -3.67 & -2.67 \\
\hline 2 & 172053.484 & -354514.90 & $5.19 \pm 0.20$ & 6290 & -5.50 & -5.83 & -5.17 \\
\hline 3 & 172053.600 & -354518.28 & $2.69 \pm 0.20$ & 3260 & -4.17 & -4.34 & -3.84 \\
\hline 4 & 172053.789 & -354513.42 & $12.82 \pm 0.18$ & 15540 & -1.68 & -2.18 & +1.14 \\
\hline 5 & 172054.052 & -354515.56 & $0.82 \pm 0.17$ & 990 & -2.18 & -2.84 & -1.84 \\
\hline 6 & 172054.055 & -354520.38 & $3.01 \pm 0.18$ & 3650 & -4.50 & -5.17 & -4.17 \\
\hline 7 & 172054.274 & -354512.11 & $1.10 \pm 0.16$ & 1330 & -7.16 & -7.66 & -6.49 \\
\hline 8 & 172054.333 & -354522.19 & $10.71 \pm 0.17$ & 12980 & -6.83 & -7.82 & -6.16 \\
\hline 9 & 172054.405 & -354522.57 & $4.39 \pm 0.17$ & 5320 & -8.49 & -9.98 & -7.99 \\
\hline 10 & 172054.419 & -354515.50 & $0.95 \pm 0.16$ & 1200 & -8.32 & -9.32 & -8.16 \\
\hline 11 & 172054.457 & -354522.57 & $83.28 \pm 0.17$ & 100900 & -7.16 & -8.16 & -6.16 \\
\hline 12 & 172054.474 & -354514.56 & $0.53 \pm 0.15$ & 640 & -1.51 & -1.84 & -0.85 \\
\hline 13 & 172054.478 & -354506.64 & $0.62 \pm 0.15$ & 750 & -1.35 & -1.84 & -1.01 \\
\hline 14 & 172054.552 & -354522.46 & $5.46 \pm 0.17$ & 6620 & -6.16 & -7.66 & -5.17 \\
\hline 15 & 172054.662 & -354543.52 & $5.10 \pm 0.37$ & 6180 & -4.83 & -5.17 & -3.67 \\
\hline 16 & 172054.838 & -354537.31 & $6.63 \pm 0.26$ & 8040 & -4.83 & -5.33 & -4.67 \\
\hline 17 & 172054.725 & -354514.22 & $18.83 \pm 0.15$ & 22820 & -5.33 & -6.00 & -3.67 \\
\hline 18 & 172054.781 & -354509.67 & $0.81 \pm 0.15$ & 980 & -2.01 & -3.01 & -1.68 \\
\hline 19 & 172054.821 & -354514.43 & $1.40 \pm 0.15$ & 1700 & -4.67 & -4.83 & -4.34 \\
\hline 20 & 172054.848 & -354536.84 & $6.11 \pm 0.25$ & 7410 & -4.34 & -4.67 & -3.84 \\
\hline 21 & 172054.881 & -354511.65 & $0.74 \pm 0.14$ & 900 & -1.68 & -2.01 & -1.35 \\
\hline 22 & 172054.921 & -354513.88 & $33.12 \pm 0.15$ & 40140 & -3.34 & -3.84 & -2.84 \\
\hline 23 & 172054.928 & -354513.60 & $12.19 \pm 0.15$ & 14770 & -5.17 & -6.49 & -4.83 \\
\hline 24 & 172054.936 & -354508.27 & $3.89 \pm 0.14$ & 4710 & -4.67 & -5.00 & -4.34 \\
\hline 25 & 172054.960 & -354513.94 & $78.63 \pm 0.15$ & 95300 & -4.34 & -4.67 & -4.00 \\
\hline 26 & 172054.984 & -35 4513.08 & .14 & 1200 & -5.17 & -5.66 & -4.83 \\
\hline 27 & 172054.991 & -354528.87 & 2.6 & 3200 & -4.50 & -4.83 & -4.17 \\
\hline 28 & 172055.009 & -354514.06 & $35.40 \pm 0.15$ & 42900 & -2.67 & -3.67 & -1.84 \\
\hline 29 & 172055.090 & -354515.54 & $4.01 \pm 0.15$ & 4860 & -2.18 & -3.17 & -1.51 \\
\hline 30 & 172055.095 & -354536.28 & $1.16 \pm 0.24$ & 1410 & -0.35 & -0.68 & -0.02 \\
\hline 31 & 172055.109 & -354536.88 & $1.86 \pm 0.25$ & 2250 & -7.16 & -7.49 & -6.66 \\
\hline 32 & 172055.165 & -354537.53 & $6.05 \pm 0.26$ & 7330 & -3.67 & -4.00 & -2.84 \\
\hline 33 & 172055.195 & -354503.81 & $1.02 \pm 0.15$ & 1240 & -0.85 & -2.01 & -0.02 \\
\hline 34 & 172055.208 & -354537.58 & $1.34 \pm 0.26$ & 1620 & -2.01 & -2.34 & -1.68 \\
\hline 35 & 172055.264 & -354536.84 & $4.79 \pm 0.25$ & 5810 & -3.84 & -4.34 & -2.67 \\
\hline 36 & 172055.251 & -354530.18 & $18.37 \pm 0.19$ & 22260 & -0.68 & -1.51 & -0.35 \\
\hline 37 & 172055.300 & -354530.15 & $1.43 \pm 0.19$ & 1730 & -1.18 & -1.68 & -1.01 \\
\hline 38 & 172055.328 & -354501.76 & $1.42 \pm 0.15$ & 1720 & -1.84 & -2.18 & -1.18 \\
\hline 39 & 172055.329 & -354505.55 & $0.60 \pm 0.15$ & 730 & -1.18 & -1.51 & -0.85 \\
\hline 40 & 172055.367 & -354505.61 & $1.37 \pm 0.15$ & 1660 & -3.34 & -4.00 & -2.84 \\
\hline 41 & 172055.392 & -354505.16 & $1.02 \pm 0.15$ & 1240 & -1.01 & -1.51 & -0.68 \\
\hline 42 & 172055.440 & -354509.78 & $11.19 \pm 0.14$ & 13560 & -4.67 & -5.33 & -4.50 \\
\hline 43 & 172055.466 & -354506.62 & & 3430 & -1.35 & -2.01 & -0.85 \\
\hline 44 & 172055.471 & -354500.86 & 0.15 & 19 & +2.64 & +1.64 & +4.80 \\
\hline 45 & 172055.513 & -354506.25 & $1.38 \pm 0.15$ & 1670 & -4.50 & -4.83 & -4.34 \\
\hline 46 & 172055.515 & -354507.64 & $1.90 \pm 0.15$ & 2300 & -5.00 & -5.50 & -4.67 \\
\hline 47 & 172055.538 & -354500.62 & $1.63 \pm 0.15$ & 1980 & -4.17 & -4.34 & -3.84 \\
\hline 48 & 172055.540 & -354509.05 & $0.74 \pm 0.14$ & 900 & -5.33 & -5.50 & -5.17 \\
\hline 49 & 172055.558 & -354526.72 & $3.32 \pm 0.18$ & 4020 & -3.34 & -3.67 & -3.01 \\
\hline 50 & 172055.581 & -354502.53 & $0.96 \pm 0.15$ & 1200 & -2.01 & -3.01 & -0.68 \\
\hline 51 & 172055.610 & -354529.45 & $5.35 \pm 0.19$ & 6480 & -2.84 & -3.17 & -2.51 \\
\hline 52 & 172055.651 & -354531.23 & $4.48 \pm 0.21$ & 5430 & -4.34 & -4.50 & -4.17 \\
\hline 53 & 172055.652 & -354501.68 & $1.68 \pm 0.15$ & 2040 & -1.18 & -2.18 & -0.02 \\
\hline 54 & 172055.656 & -354510.62 & $2.86 \pm 0.15$ & 3470 & -5.83 & -6.49 & -4.67 \\
\hline 55 & 172055.677 & -354500.39 & $296.42 \pm 0.16$ & 359260 & -2.67 & -3.17 & -1.51 \\
\hline 56 & 172055.683 & -354502.88 & $1.02 \pm 0.15$ & 1240 & -7.16 & -7.66 & -6.66 \\
\hline 57 & 172055.708 & -354511.17 & $1.42 \pm 0.15$ & 1720 & -3.51 & -3.67 & -3.17 \\
\hline 58 & 172055.771 & -354527.75 & $268.96 \pm 0.19$ & 325980 & -4.50 & -5.17 & -3.84 \\
\hline 59 & 172055.810 & -354531.07 & $2.12 \pm 0.21$ & 2570 & -5.00 & -5.66 & -4.83 \\
\hline 60 & 172055.859 & -354531.74 & $3.45 \pm 0.21$ & 4180 & -5.33 & -6.00 & -4.83 \\
\hline 61 & 172055.859 & -354528.27 & & 86 & -3.84 & -4.34 & -3.51 \\
\hline 62 & 172056.004 & -354533.04 & $4.53=$ & 5490 & -3.84 & -4.50 & -3.17 \\
\hline 63 & 172056.004 & -354523.42 & & 2400 & -3.34 & -3.67 & -3.17 \\
\hline 64 & 172056.095 & -354522.41 & $4.12 \pm 0.17$ & 4990 & -4.67 & -5.00 & -4.00 \\
\hline 65 & 172056.128 & -354523.50 & $28.48 \pm 0.18$ & 34520 & -5.00 & -5.66 & -4.50 \\
\hline 66 & 172056.164 & -354522.80 & $5.23 \pm 0.18$ & 6340 & -7.66 & -8.65 & -7.32 \\
\hline 67 & 172056.185 & -354523.85 & $10.17 \pm 0.18$ & 12330 & -7.82 & -8.82 & -6.49 \\
\hline 68 & 172056.196 & -354532.22 & $9.45 \pm 0.23$ & 11450 & -4.17 & -4.50 & -3.67 \\
\hline 69 & 172055.264 & -354536.84 & $4.80 \pm 0.25$ & 5820 & -5.33 & -5.66 & -5.00 \\
\hline 70 & 172056.285 & -354519.20 & $2.14 \pm 0.17$ & 2590 & -3.51 & -4.34 & -3.17 \\
\hline 71 & 172056.296 & -354529.73 & $43.26 \pm 0.22$ & 52430 & -5.00 & -5.83 & -4.50 \\
\hline 72 & 172056.342 & -354520.41 & $1.74 \pm 0.18$ & 2110 & -3.51 & -4.17 & -3.17 \\
\hline 73 & 172056.307 & -354526.75 & $0.90 \pm 0.20$ & 1100 & -2.18 & -2.51 & -2.01 \\
\hline 74 & 172056.341 & -354530.68 & $28.14 \pm 0.23$ & 34110 & -4.00 & -4.50 & -3.17 \\
\hline 75 & 172056.414 & -354531.72 & $171.47 \pm 0.24$ & 207820 & -3.34 & -3.67 & -3.01 \\
\hline 76 & 172056.423 & -354529.69 & $9.90 \pm 0.23$ & 12000 & -4.00 & -6.00 & -3.67 \\
\hline 77 & 172056.488 & -354530.49 & $5.38 \pm 0.24$ & 6520 & -2.51 & -3.67 & -2.01 \\
\hline 78 & 172056.708 & -354532.61 & $13.34 \pm 0.28$ & 16170 & -2.51 & -3.01 & -1.18 \\
\hline 79 & 172057.028 & -354535.92 & $2.02 \pm 0.37$ & 2450 & -5.33 & -5.66 & -4.34 \\
\hline 80 & 172056.903 & -354524.93 & $2.06+0.24$ & 2500 & -3.51 & -3.84 & -3.17 \\
\hline
\end{tabular}


TABLE 5

1.3 MILLIMETER SPECTRAL LINES DETECTED TOWARD SMA1

\begin{tabular}{|c|c|c|c|c|}
\hline Species & Transition & Frequency & $E_{\text {lower }}\left(\mathrm{cm}^{-1}\right)^{\mathrm{a}}$ & $T_{\text {Brightness }}(\mathrm{K})^{\mathrm{b}}$ \\
\hline $\mathrm{H}_{2} \mathrm{~S}$ & $2_{2,0}-2_{1,1}$ & 216.710435 & 51.1 & 13 \\
\hline $\mathrm{HCOOCH}_{3}-\mathrm{E}$ & $18_{4,15}-17_{4,14}$ & 216.830112 & 66.2 & 6.2 \\
\hline $\mathrm{HCOOCH}_{3}-\mathrm{A}$ & $18_{2,16}-17_{2,15}$ & 216.838846 & 66.2 & 4.8 \\
\hline $\mathrm{C}_{2} \mathrm{H}_{3} \mathrm{CN}$ & $23_{2,22}-22,2,21$ & 216.936717 & 85.9 & 0.8 \\
\hline $\mathrm{CH}_{3} \mathrm{OH}-\mathrm{E}$ & $5_{+1,4}-4+2,2$ & 216.945600 & 31.6 & 11 \\
\hline $\mathrm{HCOOCH}_{3}-\mathrm{E}$ & $20_{1,20}-19_{1,19}$ & 216.964789 & 70.3 & 10 \\
\hline $\mathrm{HCOOCH}_{3}-\mathrm{A}$ & $20_{1,20}-19_{1,19}$ & 216.965991 & 70.3 & \multirow{2}{*}{\} 13} \\
\hline $\mathrm{HCOOCH}_{3}-\mathrm{E}$ & $20_{2,19}-19_{0,19}$ & 216.966247 & 70.3 & \\
\hline $\mathrm{HCOOCH}_{3}-\mathrm{A}$ & $20_{0,20}-19_{0,19}$ & 216.967334 & 70.3 & 11 \\
\hline Unidentified & $\ldots$ & 217.020 & $\ldots$ & 2.3 \\
\hline $\mathrm{SiO}$ & $5-4$ & 217.104980 & 14.5 & 2.8 \\
\hline $\mathrm{CH}_{3} \mathrm{OCH}_{3}$ & $36_{0,36,1}-36_{1,35,1}$ & 217.168120 & 436.6 & 1.2 \\
\hline $\mathrm{CH}_{3} \mathrm{OCH}_{3}$ & $22_{4,19,2}-22_{3,20,2}$ & 217.189667 & 168.9 & \multirow{2}{*}{2.9} \\
\hline $\mathrm{CH}_{3} \mathrm{OCH}_{3}$ & $22_{4,19,3}-22_{3,20,3}$ & 217.189667 & 168.9 & \\
\hline $\mathrm{CH}_{3} \mathrm{OCH}_{3}$ & $22_{4,19,1}-22_{3,20,1}$ & 217.191424 & 168.9 & 4.8 \\
\hline $\mathrm{CH}_{3} \mathrm{OCH}_{3}$ & $22_{4,19,0}-22_{3,20,0}$ & 217.193170 & 168.9 & 3.6 \\
\hline DCN & $3-2^{\mathrm{c}}$ & 217.238630 & 7.2 & 5.7 \\
\hline $\mathrm{CH}_{3} \mathrm{OH}-\mathrm{A}^{-}\left(v_{t}=1\right)$ & $6_{1,5}-7_{2,6}$ & 217.299202 & 252.6 & 8.4 \\
\hline Unidentified & $\ldots$ & 217.312 & $\ldots$ & 2.2 \\
\hline${ }^{13} \mathrm{CH}_{3} \mathrm{OH}-\mathrm{A}^{+}$ & $10_{2,8}-9_{3,7}$ & 217.399550 & 105.6 & 2.4 \\
\hline Unidentified & $\ldots$ & 217.418 & $\cdots$ & 1.9 \\
\hline $\mathrm{CH}_{3} \mathrm{OH}-\mathrm{A}^{+}\left(v_{t}=1\right)$ & $15_{6,10}-16_{5,11}$ & 217.642859 & 511.0 & 3.3 \\
\hline $\mathrm{CH}_{3} \mathrm{OH}-\mathrm{E}$ & $20+1,19-20+0,20$ & 217.886390 & 346.1 & 5.0 \\
\hline Unidentified & $\ldots$ & 217.959 & $\ldots$ & 1.9 \\
\hline $\mathrm{OC}^{13} \mathrm{~S}$ & $18-17$ & 218.198998 & 61.9 & 3.7 \\
\hline $\mathrm{H}_{2} \mathrm{CO}$ & $3_{0,3}-2_{0,2}$ & 218.222192 & 7.3 & 8.4 \\
\hline $\mathrm{HCOOCH}_{3}-\mathrm{E}$ & $17_{1,16}-16_{1,15}$ & 218.280835 & 62.0 & 4.4 \\
\hline $\mathrm{HCOOCH}_{3}-\mathrm{A}$ & $17_{3,14}-16_{3,13}$ & 218.297831 & 62.0 & 4.9 \\
\hline $\mathrm{HC}_{3} \mathrm{~N}$ & $24-23^{\mathrm{d}}$ & 218.324723 & 83.8 & 21 \\
\hline $\mathrm{C}_{2} \mathrm{H}_{5} \mathrm{CN}$ & $24_{3,21}-23_{3,20}$ & 218.389970 & 90.0 & 7.7 \\
\hline $\mathrm{CH}_{3} \mathrm{OH}-\mathrm{E}$ & $4+2,2-3+1,2$ & 218.440040 & 24.3 & 23 \\
\hline $\mathrm{C}_{2} \mathrm{H}_{3} \mathrm{CN}$ & $23_{5,19}-22_{5,18}$ & 218.451298 & 117.7 & 2.7 \\
\hline $\mathrm{C}_{2} \mathrm{H}_{3} \mathrm{CN}$ & $23_{5,18}-225,17$ & 218.452357 & 117.7 & 2.5 \\
\hline $\mathrm{NH}_{2} \mathrm{CHO}$ & $10_{1,9}-9_{1,8}$ & 218.459653 & 35.0 & 3.4 \\
\hline $\mathrm{H}_{2} \mathrm{CO}$ & $3_{2,2}-22,1$ & 218.475632 & 40.4 & 7.7 \\
\hline $\mathrm{CH}_{3} \mathrm{OCH}_{3}$ & $23_{3,21,1}-23_{2,22,1}$ & 218.492412 & 176.1 & 4.7 \\
\hline $\mathrm{C}_{2} \mathrm{H}_{3} \mathrm{CN}$ & $23_{4,20}-22_{4,19}$ & 218.573646 & 104.2 & 1.7 \\
\hline $\mathrm{C}_{2} \mathrm{H}_{3} \mathrm{CN}$ & $23_{3,21}-22_{3,20}$ & 218.585072 & 93.7 & 1.6 \\
\hline $\mathrm{CN}$ & $2_{0,2,3}-1_{0,1,2}$ & 226.659558 & 3.8 & -3.4 \\
\hline $\mathrm{CN}$ & $2_{0,2,1}-1_{0,1,1}$ & 226.663693 & 3.8 & -0.9 \\
\hline $\mathrm{CN}$ & $2_{0,2,2}-1_{0,1,1}$ & 226.679311 & 3.8 & -4.9 \\
\hline $\mathrm{HCOOCH}_{3}-\mathrm{E}$ & $20_{2,18}-19_{2,18}$ & 226.713060 & 76.0 & 5.8 \\
\hline $\mathrm{HCOOCH}_{3}-\mathrm{A}$ & $20_{2,19}-19_{2,18}$ & 226.718688 & 76.0 & 5.3 \\
\hline $\mathrm{HCOOCH}_{3}-\mathrm{E}$ & $20_{3,18}-19_{3,17}$ & 226.773130 & 76.0 & 5.2 \\
\hline $\mathrm{HCOOCH}_{3}-\mathrm{A}$ & $20_{1,19}-19_{1,18}$ & 226.778707 & 76.0 & 6.0 \\
\hline $\mathrm{CN}$ & $2_{0,3,3}-1_{0,2,2}$ & 226.874191 & 3.8 & \multirow{2}{*}{\}-6.9} \\
\hline $\mathrm{CN}$ & $2_{0,3,4}-1_{0,2,3}$ & 226.874781 & 3.8 & \\
\hline $\mathrm{CN}$ & $2_{0,3,2}-1_{0,2,1}$ & 226.875896 & 3.8 & -6.1 \\
\hline $\mathrm{CN}$ & $2_{0,3,2}-1_{0,2,2}$ & 226.887420 & 3.8 & -2.2 \\
\hline $\mathrm{CN}$ & $20,3,3-1,2,3$ & 226.892128 & 3.8 & -1.5 \\
\hline $\mathrm{HCOOCH}_{3}-\mathrm{E}$ & $194,16-184,15$ & 227.019490 & 73.5 & 4.8 \\
\hline $\mathrm{HCOOCH}_{3}-\mathrm{A}$ & $19_{2,17}-18_{2,16}$ & 227.028070 & 73.4 & 5.0 \\
\hline $\mathrm{CH}_{3} \mathrm{OH}-\mathrm{E}$ & $21_{+1,20}-21_{+0,21}$ & 227.094601 & 379.6 & 6.9 \\
\hline Unidentified & $\ldots$ & 227.126 & $\ldots$ & 2.2 \\
\hline Unidentified & $\ldots$ & 227.170 & $\ldots$ & 2.0 \\
\hline Unidentified & $\ldots$ & 227.221 & $\ldots$ & 1.8 \\
\hline $\mathrm{CH}_{3} \mathrm{OH}-\mathrm{E}$ & $12_{-1,12}-11_{+2,9}$ & 227.229600 & 122.0 & 2.2 \\
\hline $\mathrm{g}-\mathrm{C}_{2} \mathrm{H}_{5} \mathrm{OH}$ & $13_{3,10,1}-12_{3,9,1}$ & 227.294752 & 95.7 & 1.8 \\
\hline${ }^{13} C^{34} S$ & $5-4$ & 227.300506 & 15.16 & 3.9 \\
\hline $\mathrm{HC}_{3} \mathrm{~N}$ & $25-24^{\mathrm{d}}$ & 227.418905 & 91.03 & 17 \\
\hline $\mathrm{HCOOCH}_{3}-\mathrm{E}$ & $21_{1,21}-20_{1,20}$ & 227.560955 & 77.5 & 12 \\
\hline $\mathrm{HCOOCH}_{3}-\mathrm{E}$ & $21_{2,20}-20_{2,19}$ & 227.561753 & 77.5 & \multirow{2}{*}{\} 12} \\
\hline $\mathrm{HCOOCH}_{3}-\mathrm{A}$ & $21_{1,21}-20_{1,20}$ & 227.561944 & 77.5 & \\
\hline $\mathrm{HCOOCH}_{3}-\mathrm{A}$ & $21_{0,21}-20_{0,20}$ & 227.562740 & 77.5 & \multirow{3}{*}{$\begin{array}{l}10 \\
1.5\end{array}$} \\
\hline $\mathrm{t}-\mathrm{C}_{2} \mathrm{H}_{5} \mathrm{OH}$ & $18_{5,13,2}-18_{4,14,2}$ & 227.606079 & 114.3 & \\
\hline $\begin{array}{l}\mathrm{NH}_{2} \mathrm{CHO} \\
\mathrm{CH}_{3} \mathrm{OCH}_{3}\end{array}$ & $\begin{array}{c}11_{0,11}-10_{0,10} \\
26_{5,21,0}-26_{4,22,0}\end{array}$ & $\begin{array}{l}227.606176 \\
227.647849\end{array}$ & $\begin{array}{c}38.5 \\
239.7\end{array}$ & \\
\hline $\mathrm{CH}_{3} \mathrm{OCH}_{3}$ & $26_{5,21,1}-26_{4,22,1}$ & 227.647984 & 239.7 & 4.5 \\
\hline $\mathrm{CH}_{3} \mathrm{OCH}_{3}$ & $26_{5,21,2}-26_{4,22,2}$ & 227.648120 & 239.7 & \multirow{3}{*}{\} 4.6} \\
\hline $\mathrm{CH}_{3} \mathrm{OCH}_{3}$ & $24_{3,22,2}-24_{2,23,2}$ & 227.654386 & 190.9 & \\
\hline $\mathrm{CH}_{3} \mathrm{OCH}_{3}$ & $24_{3,22,3}-24_{2,23,3}$ & 227.654386 & 190.9 & \\
\hline $\mathrm{CH}_{3} \mathrm{OCH}_{3}$ & $24_{3,22,1}-24_{2,23,1}$ & 227.657025 & 190.9 & 4.9 \\
\hline $\mathrm{CH}_{3} \mathrm{OCH}_{3}$ & $24_{3,22,0}-24_{2,23,0}$ & 227.659664 & 190.9 & 2.0 \\
\hline $\mathrm{C}_{2} \mathrm{H}_{5} \mathrm{CN}$ & $25_{3,22}-24_{3,21}$ & 227.780972 & 97.3 & 6.1 \\
\hline $\mathrm{CH}_{3} \mathrm{OH}-\mathrm{A}^{+}$ & $16_{1,16}-15_{2,13}$ & 227.814650 & 219.8 & 9.5 \\
\hline $\mathrm{g}-\mathrm{C}_{2} \mathrm{H}_{5} \mathrm{OH}$ & $13_{1,12,1}-12_{1,11,1}$ & 227.891911 & 89.7 & 0.9 \\
\hline
\end{tabular}


TABLE 6

DERIVED 1.3 MILLIMETER CONTINUUM PROPERTIES

\begin{tabular}{lcccc}
\hline \hline Source & $\begin{array}{c}T_{\text {dust }} \\
(\mathrm{K})\end{array}$ & $\begin{array}{c}M_{\text {gas }} \\
\left(\mathrm{M}_{\odot}\right)\end{array}$ & $\begin{array}{c}N_{\mathrm{H}_{2}} \times 10^{23} \\
\left(\mathrm{~cm}^{-2}\right)\end{array}$ & $\begin{array}{c}n_{\mathrm{H}_{2}} \times 10^{7} \\
\left(\mathrm{~cm}^{-3}\right)\end{array}$ \\
\hline SMA1 & $155-165$ & $16-14$ & $7.7-6.8$ & $1.3-1.2$ \\
SMA2 & $135-155$ & $7-6$ & $6.4-5.7$ & $1.6-1.4$ \\
SMA3 & $20-50$ & $74-23$ & $40-12$ & $7-2$ \\
SMA4 & $20-50$ & $52-16$ & $30-9$ & $6-2$ \\
SMA5 & $20-50$ & $28-9$ & $18-6$ & $4-1$ \\
SMA6 & $25-50$ & $32-13$ & $58-17$ & $16-5$ \\
SMA7 & $20-50$ & $22-7$ & $8-3$ & $1-0.4$ \\
& & & & \\
\hline
\end{tabular}

